ORIGINAL VERSION SUBMITTED to APJ LETTERS ON 21 DECEMBER 2012. This VERSION ACCEPTED ON 7 OCTOBER 2014.

Preprint typeset using LTTEX style emulateapj v. 5/2/11

\title{
LUMINOUS BLUE VARIABLES AND SUPERLUMINOUS SUPERNOVAE FROM BINARY MERGERS
}

\author{
STEPHEN JUSTHAM \\ The Key Laboratory of Optical Astronomy, National Astronomical Observatories, The Chinese Academy of Sciences, Datun Road, Beijing 100012, China \\ previously at: \\ The Kavli Institute for Astronomy and Astrophysics, Peking University, Beijing 100871, China
}

PHILIPP PODSIADLOWSKI

Astrophysics Sub-Department, University of Oxford, Oxford OX1 3RH, United Kingdom

\author{
JORICK S. VINK \\ Armagh Observatory, College Hill, Armagh BT61 9DG, Northern Ireland \\ Original version submitted to ApJ Letters on 21 December 2012. This version accepted on 7 October 2014.
}

\begin{abstract}
Evidence suggests that the direct progenitor stars of some core-collapse supernovae (CCSNe) are luminous blue variables (LBVs), perhaps including some 'superluminous supernovae' (SLSNe). We examine models in which massive stars gain mass soon after the end of core hydrogen burning. These are mainly intended to represent mergers following a brief contact phase during early Case B mass transfer, but may also represent stars which gain mass in the Hertzsprung Gap or extremely late during the main-sequence phase for other reasons. The post-accretion stars spend their core helium-burning phase as blue supergiants (BSGs), and many examples are consistent with being LBVs at the time of core collapse. Other examples are yellow supergiants at explosion. We also investigate whether such post-accretion stars may explode successfully after core collapse. The final core properties of post-accretion models are broadly similar to those of single stars with the same initial mass as the pre-merger primary star. More surprisingly, when early Case B accretion does affect the final core properties, the effect appears likely to favour a successful SN explosion, i.e., to make the core properties more like those of a lower-mass single star. However, the detailed structures of these cores sometimes display qualitative differences to any single-star model we have calculated. The rate of appropriate binary mergers may match the rate of SNe with immediate LBV progenitors; for moderately optimistic assumptions we estimate that the progenitor birthrate is $\sim 1 \%$ of the CCSN rate.
\end{abstract}

Subject headings: binaries: close — supernovae: general

\section{INTRODUCTION}

Supernovae ( $\mathrm{SNe})$ - explosions of stars - have long been studied as complex physical systems which play a vital role in shaping the composition and structure of the Universe. Despite the extensive history of the field, recent discoveries have challenged some strongly-held expectations. One major surprise was that some massive stars appear to explode during a phase where they appear as "luminous blue variables" (LBVs): observations of radio modulations from some $\mathrm{SNe}$ indicated that the SN ejecta interacted with circumstellar material (CSM) similar to that found around LBVs undergoing $\mathrm{S}$ Doradus cycles, during which envelope material is ejected episodically (Kotak \& Vink 2006). This interpretation was strengthened by observations of multiple P Cygni absorption profiles in the spectrum of the interacting SN 2005gj (Trundle et al.2008), which provided evidence for multiple shells with characteristic LBV wind velocities, possibly associated with multiple LBV outbursts; Kiewe et al. (2012) subsequently found P Cygni absorption features in four additional SN IIn. Further support for this inference was provided when the immediate progenitor of the type IIn SN 2005gl was identified as having been a very luminous star, consistent with an LBV (Gal-Yam et al. 2007), Gal-Yam \& Leonard 2009).

Perhaps the strongest evidence yet known in favour of LBVlike outbursts from SN progenitors comes from the systems

SJ: sjustham@bao.ac.cn; PhP: podsi@astro.ox.ac.uk which produced SN 2009ip and SN 2010mc, both of which were classified as Type IIn events. Ofek et al. (2013b) found that the progenitor of SN 2010mc ejected $\sim 10^{-2} M_{\odot}$ during an outburst only 40 days before it exploded as a SN. The system which produced SN 2009ip has displayed several outbursts since an outbursting LBV was first identified at that location (Maza et al. 2009, Miller et al. 2009a; Berger et al. 2009, Li et al. 2009). The most recent outburst from SN 2009ip may well mark the final explosion of the star (Mauerhan et al. 2013, Smith et al. 2014), although there is some uncertainty over whether the outburst was terminal (Fraser et al.2013; Margutti et al.2014).

One complication which arises when interpreting this observational evidence in terms of understanding SN progenitors is that the class of LBVs is poorly understood and inhomogeneous. In broad terms, LBVs are massive, hot stars located near the Eddington limit, and are subject to occasional outbursts accompanied by mass ejection (see, e.g., Vink 2012). However, the standard S Doradus-type LBV outbursts are dissimilar from events such as the Great Eruption of Eta Carinae. Hence, even though Eta Carinae is referred to as an LBV, it and other objects which produce similar rare giant eruptions may arise from a different mechanism than canonical S Dor LBVs. Furthermore, even though the phenomenology of typical LBV outbursts is fairly well established (Humphreys \& Davidson 1994), the specific physical mechanism responsible for even those S Dor LBV mass-loss 
events is unclear. This uncertainty remains despite a great deal of theoretical attention, although there is broad agreement that high stellar luminosities - near to the Eddington limit - could enable S Dor-type instabilities (see, e.g., Joss et al. 1973; Glatzel \& Kiriakidis 1993; Langer 1998; Shaviv 2001;:Vink \& de Koter 2002; Smith \& Owocki 2006, Gräfener et al. 2012; Guzik \& Lovekin 2014; Owocki 2014). As a result, we later compare our models to the empirical position of the S Dor instability strip on the HR diagram (see, e.g., Groh et al.2009), not to theoretical models for LBV instabilities.

The term "LBV" thus defines a broad phenomenology rather than an evolutionary stage, and the evolutionary nature of LBVs is as yet unknown. Nonetheless, canonical LBVs were not generally expected to be immediate progenitors of core-collapse SNe. Standard stellar evolution theory predicts that single massive stars which become LBVs do so near the end or after the completion of core hydrogen $(\mathrm{H})$ burning, then typically lose their H-rich envelopes in the LBV phase and become H-deficient Wolf-Rayet stars, where they spend several $10^{5} \mathrm{yr}$ burning helium (He) in the core before they explode in a core-collapse SN (CCSN), long after they have passed through the LBV phase (for a review see, e.g., Langer 2012).

A further challenge when trying to understand the population of these SNe with apparent LBV progenitors is that the LBV-SNe are unlikely to correspond with all members of one of the phenomenological SN types. SNe with presumed LBV progenitors are of type IIn or IIb, and some previous work has argued that all type IIn SNe may be generated by LBVlike progenitors (see, e.g., Gal-Yam et al. 2007; Kiewe et al. 2012). However, the type IIn SN phenomenon can potentially be produced by a heterogeneous set of circumstances (Kotak et al. 2004), as demonstrated by events such as SN 2002ic and SN 2005gj (Hamuy et al. 2003, Aldering et al. 2006). Those SNe showed strong circumstellar interaction like that seen in type IIn SNe, but each is thought to have been powered by a type Ia SN, not a CCSN. SN PTF11kx showed similar, but somewhat weaker interaction (Dilday et al. 2012), which suggests that there may be a continuum of $\mathrm{H}$ emission line strengths arising from type Ia SN progenitors. Many other $\mathrm{SNe}$ which were classified as IIn could easily have been disguised SNe Ia. This suspicion was strengthened by Anderson et al. (2012), who found that their sample of SNe exhibiting type IIn phenomenology shows less clear association with star formation than any other SN sub-type generally attributed to core collapse. Based on their data, they suggested that the majority of IIn SNe arise from relatively low-mass progenitors, i.e., are not associated with LBVs (see also Habergham et al. 2014). There may be tension between this conclusion and the inference that the majority of SN IIn display preexplosion outbursts (Ofek et al.2014), if those pre-explosion events are shown to be LBV outbursts from the star which explodes. Nonetheless, the evidence that most SN which could be classified as belonging to "Type IIn" may not arise from LBVs does not affect the evidence that some SNe have direct LBV progenitors. However, this heterogeneity is a complication when trying to determine the rate at which LBVs are formed and explode.

\section{1. "Superluminous" supernovae from LBV progenitors?}

The interest surrounding LBVs as immediate SN progenitors increased further following the discovery of the extraordinarily luminous type IIn SN 2006gy (Ofek et al. 2007; Smith et al. 2007), which was also suggested to have been produced by an LBV star. Numerous similarly outstanding events have since been identified (Quimby et al. 2007; Smith et al. 2008; Gezari et al.2009, Miller et al.2009b; Drake et al. 2010; GalYam 2012). In this work, we mainly consider a subset of these 'superluminous' SNe (SLSNe), the H-rich 'SLSN-II' (for a review, see Gal-Yam 2012). One popular explanation for the high luminosity of these $\mathrm{SNe}$ is that a standard amount of CCSN energy input is radiated away far more efficiently than in a canonical CCSN. This is believed to be due to interaction of the SN ejecta with a dense CSM, which causes rapid deceleration of the $\mathrm{SN}$ shock, thereby converting kinetic energy into radiation (see, e.g., Smith et al. 2007; Ofek et al. 2007; Smith \& McCray 2007; van Marle et al.|2010; Gal-Yam 2012. although Moriya et al. 2013 found it challening to reproduce the SN 2006gy light-curve using CSM interaction). Prior to the interaction model for SN 2006gy, several other SNe had shown evidence for large amounts of mass ejection soon before the explosion (Dopita et al. 1984; Chugai \& Danziger 1994, Chugai et al. 2004). As LBVs are a class of single stars which experience phases of mass loss drastic enough to account for the required CSM densities, they have widely been regarded as potential progenitors of SLSNe. The properties of LBV ejections before collapse might control the CSM densities at the time of explosion, and thereby the radiative efficiency of the SN. For example, the timing of the last pre-SN LBV outburst may need to be sufficiently close to the SN to lead to a SLSN, or the amount of mass ejected may need to be unusually large (at one extreme, a giant eruption may be required). Hence similar stellar systems might explain both the LBV-SNe with normal luminosity and the relevant subset of SLSNe, separated only by random variations in the pre-SN outburst properties.

Nevertheless, a key issue with single-star LBVs as SN progenitors - both with normal and exceptionally high luminosity - is that stars in the appropriate mass range are typically expected to produce faint $\mathrm{SNe}$ (if core collapse leads to any $\mathrm{SN}$ explosion at all). The reason is that they are predicted to quietly form black holes, without the strong outward-moving shock required for a typical SN explosion energy (see, e.g., Fryer 1999, Heger et al. 2003; for an observational perspective see, e.g., Kochanek et al. 2008, Kochanek 2014). There are likely exceptions to this statement, e.g., the "collapsars" which are a consequence of rapid-rotation in the core at core collapse (Woosley 1993; MacFadyen \& Woosley 1999), but those are thought to be extremely rare events.

\subsection{Non-LBV models for unusually luminous SNe and for pre-core-collapse mass ejection}

LBV outbursts are not the only way to eject substantial amount of mass from the stellar envelope. The type of binary interaction known as common-envelope (CE) evolution may also do the trick. Hence an alternative possibility for the presence of a massive CSM close to an exploding star is the recent ejection of a CE in a massive binary system (Ofek et al.2007, Chevalier 2012). At least some CCSNe are predicted to occur during such phases (Podsiadlowski et al.1990), although it is unclear whether the empirical event rates could be matched without fine-tuning. Note that canonical CE ejection may not be necessary to eject sufficient mass to produce SLSNe, as mergers may also eject significant amounts of mass (see, e.g., Podsiadlowski et al. 1991, Podsiadlowski 1992, Morris \& Podsiadlowski 2007, 2009, Ivanova et al.2013). A related model is that of Soker \& Kashi (2013), which aims to explain both the pre-SN outburst of SN 2009ip and the CSM through a particular binary merger scenario. In addition, Mackey et al. 
(2014) have suggested that external photoionization may be able to trap the normal winds of red supergiant stars sufficiently well to explain $\mathrm{SNe}$ which show evidence of interaction with CSM.

Extremely massive stars are predicted to produce pairinstability $\mathrm{SNe}$ (PISNe). The mass limit is generally considered to be in excess of $\approx 150 M_{\odot}$ (somewhat dependent on metallicity and other assumptions; see, e.g., Barkat et al. 1967, Rakavy \& Shaviv 1967; Heger et al. 2003), although mixing due to rapid rotation might significantly lower that limit (Chatzopoulos \& Wheeler 2012a). The likely appearance of such $\mathrm{SNe}$ has recently been studied by, e.g., Kasen et al. (2011) and Kozyreva et al. (2014). However, there is no reason to believe that such events have been confused with the cases which we seek to explain. Strong candidates for PISNe have been identified (Gal-Yam et al. 2009, GalYam 2012), although other models have also been proposed (see, e.g., Moriya et al. 2010a; Nicholl et al. 2013). Slightly less massive stars are expected to produce pulsational PISNe, which provide yet another plausible explanation for the CSM around these SN progenitors at the time of explosion (for which, see Woosley et al. 2007, Waldman 2008, Chatzopoulos \& Wheeler 2012b, Chen et al. 2014). Models have also predicted that the envelopes of some luminous red supergiant (RSG) SN progenitors could produce pulsation-driven superwinds towards the end of their life (see, e.g., Heger et al. 1997, Yoon \& Cantiello 2010). Arguments have even been made in favour of mass-loss driven by the very late nuclear evolution of the stellar core (Quataert \& Shiode 2012; Shiode \& Quataert 2014; Moriya 2014). For the specific case of SN 2009ip, Ofek et al. (2013a) argue that their measurements of the CSM density are more consistent with a model like that of Quataert \& Shiode (2012) than ejections from pulsational pair-instability. All of these late mass-loss mechanisms could naturally explain why some core-collapse SNe occur in denser-than-otherwise-expected CSM. Nonetheless, the models which require RSGs could clearly not explain SNe with BSG progenitors (and models which require normal core collapse from BSGs are incomplete without an explanation for why the stars reach core collapse as BSGs).

A further class of models for some SLSNe invoke the rapid spin-down of a magnetar to power the luminosity (see, e.g., Kasen \& Bildsten 2010, Woosley 2010), which have been particularly successful in fitting the lightcurves of some extremely luminous H-poor SNe (Inserra et al. 2013; Howell et al. 2013).

Obviously more than one of the proposed models may work, and even the subset of "SLSN-II" might have heterogenous origins.

\subsection{Models for blue supergiant $S N$ progenitors}

Models for direct SN progenitors which are blue supergiants (BSGs) at core collapse have existed for many years, but only for cases where the BSG is not an LBV. The most well-known example of a BSG progenitor is that of $\mathrm{SN}$ 1987A. This paper examines whether a binary merger model, a variation of a previous model for SN 1987A (Podsiadlowski 1992) is able to explain LBV-SN progenitors. That merger model is not only able to explain the BSG progenitor of SN 1987A, but it also provides a natural explanation for the distinctive circumstellar structures seen in the remnant of SN 1987A (Podsiadlowski et al. 1991; Podsiadlowski 1992 . Morris \& Podsiadlowski 2007, 2009). Here we extend that work and demonstrate that more massive mergers are capable of producing SN progenitors which are plausibly luminous enough to be LBVs at the time of explosion.

We suggest that LBV-SN progenitors can form from massive binary systems which merge soon after the more massive star has finished core $\mathrm{H}$ burning, i.e., as it is expanding across the Hertzsprung Gap (HG). The fact that such a star could gain mass is somewhat counter-intuitive, but it has been previously studied and accepted as at least plausible; we explain the merger mechanism below. Our calculations could also apply to other situations in which massive stars gain mass soon after the end of the main sequence (i.e."early Case B" accretion; see also Podsiadlowski et al. 1992). Podsiadlowski \& Joss (1989) and Braun \& Langer (1995) previously studied how accretion onto massive main-sequence stars might produce BSG SN progenitors; in this respect this paper also extends that work, although stable mass-transfer onto a HG star is probably far less common than accretion via mergers (as we discuss in $\$ 5.2$. . Roughly coincident with submission of the original version of this work another paper was submitted which addresses similar possibilities (Vanbeveren et al. 2013). Glebbeek et al. (2013) also published work with similar aspects.

Binary interactions are expected to have a significant effect on the lives of a large fraction of massive stars, probably the majority of them, with observational studies of massive stars concluding that most massive stars occur in interacting binary systems (see, e.g., Abt \& Levy 1976, 1978, Kobulnicky \& Fryer 2007, Eggleton \& Tokovinin 2008, Sana et al. 2012, 2013). Close binary systems have long been argued to be responsible for much of the diversity of observed SN types (Podsiadlowski et al. 1992), and stellar mergers may well explain all of the B[e] supergiants (Podsiadlowski et al. 2006). Indeed, mergers are expected to be so common that de Mink et al. (2014) predicted that $8_{-4}^{+9} \%$ of observed early-type stars are merger products.

In contrast to models for the production of BSG and LBV SNe via binary interactions or stellar collisions, Groh et al. (2013) have argued that the larger He cores produced by stellar rotation allow some single stars to appear as LBVs at the time of explosion (based on evolutionary calculations from Ekström et al. 2012). Their proposed LBV-SN progenitors have initial masses of 20 and $25 M_{\odot}$, with respective final masses of 7.1 and $9.6 M_{\odot}$ (and surface He mass fractions of 0.74 and 0.9 ) following significant mass loss in an RSG phase. At explosion these stellar models retain little $\mathrm{H}$; Groh et al. state that the He-rich core accounts for $94 \%$ and $100 \%$ of the stellar mass respectively, which allows minimal room for additional mass loss via LBV outbursts before explosion without significantly affecting their surface properties.

Evidence in favour of the idea that some LBVs are produced following binary mergers arises from the observations that LBVs tend to be rapidly rotating (Groh et al. 2009) and that they also have a lower binary fraction than otherwise similar massive stars (see, e.g., Vink 2012; however, whilst Smith \& Tombleson 2014 agree that LBVs are likely to have gained mass from a companion, they argue in favour of stable mass transfer rather than mergers). Rapid rotation may well promote LBV instabilities (Langer 1997, 1998). However, although observed LBVs are rapidly rotating, it does not automatically follow that any additional mixing due to rapid rotation is important for explaining their properties. For the model which we present, the merger (or accretion) occurs during the $\mathrm{HG}$, i.e., after strong molecular-weight gradients have 
been generated within the star. It has previously been shown that rotational mixing is extremely unlikely to occur across those gradients, and therefore unlikely to significantly affect the evolution of stars produced by our proposed scenario (see, e.g., Mestel 1953, 1957, Mestel \& Moss 1986). Previous authors have argued that Case A mergers of massive stars could produce sufficient rotationally-driven mixing to cause homogeneous evolution and thereby produce the progenitors of long-duration gamma-ray bursts (see, e.g., Woosley \& Heger 2006, de Mink et al. 2013, along with related work on rapidlyrotating single stars by Yoon \& Langer 2005; Yoon et al. 2006). It may be that early Case A mergers, which occur before large composition gradients have been generated, can produce stars in which rotational mixing dominates their future evolution, but because of the molecular weight gradients such an outcome is less likely for early Case B mergers. Late Case A mergers, or mergers of Case A contact binaries which occur after the primary evolves off the main sequence, may also have sufficiently well-developed composition profiles to stabilise them against rotational mixing.

\subsection{The diversity of merger mechanisms}

There are multiple potential causes for stellar mergers. Perhaps the best-known binary merger scenario occurs for binary systems which are unstable to mass transfer on a dynamical timescale. For stars with radiative envelopes, this is thought to occur only when the donor star is a factor of a few more massive than the accretor (see, e.g., Hjellming \& Webbink 1987). Those high-mass-ratio mergers would only allow primary stars to increase their mass by a correspondingly small fraction. Mergers following such an instability would thus not allow stars which are typically expected to produce a neutron star - i.e., those with an initial main-sequence mass of $\approx 25 M_{\odot}$ or less - to become massive enough to produce LBVs (unless the mass limit for LBVs is significantly below $\left.35 M_{\odot}\right)$.

However, there is an alternative merger mechanism that can produce the LBV-SN progenitors which this paper aims to explain. These mergers would occur as the primary star is expanding away from the main sequence. It is well established that some massive binary systems which transfer mass early in the HG can enter into a contact phase. The reason for this is that the accreting star is forced to swell up, since the accretion timescale is much shorter than the thermal timescale of its envelope. This is expected to occur when the donor is at least 25\% more massive than the accretor (Pols 1994), although there is some subtlety in how the precise timing of the mass-transfer phase affects the formation of contact (Wellstein et al. 2001). Many of the systems which enter contact should then merge, though it is difficult to be precise about how large a fraction will do so (Podsiadlowski 2010). We discuss the likely rates in $\$ 5$. Even though the occurrence rate of that merger mechanism is not precisely known, we consider its existence to be relatively robust. Fig.1 presents a schematic of the scenario, along with a labelled example of a potential binary evolution.

In addition to that early Case B binary merger mechanism, some Case A massive contact binaries likely become unstable and merge after the primary starts to expand at the end of the main sequence. The structure and evolution of contact binaries is one of the areas of stellar evolution which is extremely poorly understood, and therefore we only consider them briefly in the rest of the paper. Nonetheless, some of these systems might increase the rate of LBV-SN progenitors
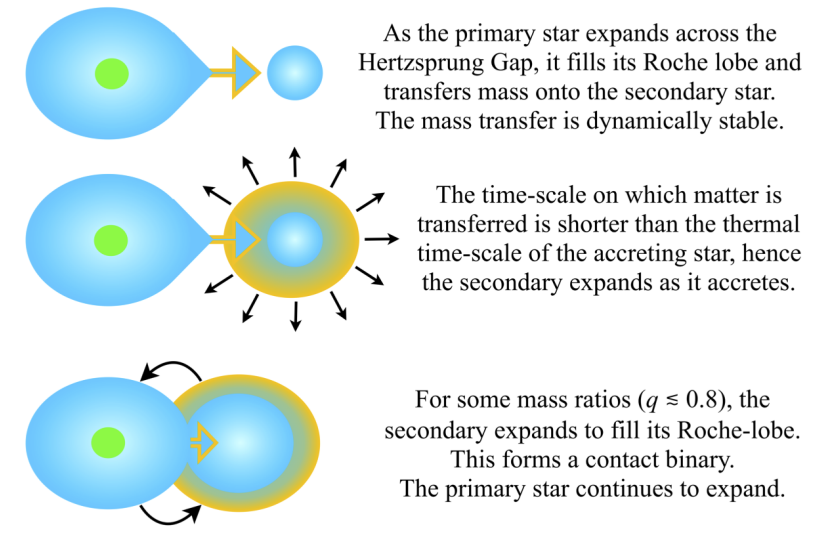

For some mass ratios $(q \leqslant 0.8)$, the secondary expands to fill its Roche-lobe. This forms a contact binary.

The primary star continues to expand.
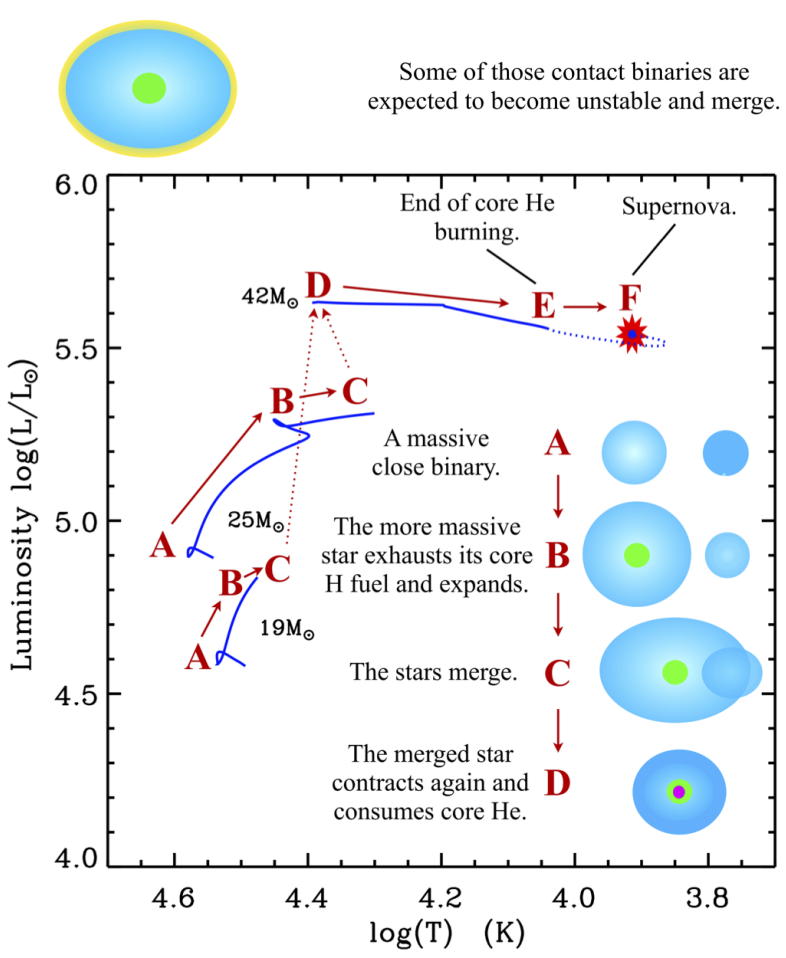

Figure 1. Schematic outline of the merger scenario which can lead to LBVSN progenitors. The top panel illustrates a mechanism through which dynamically stable mass transfer leads to a merger via a contact phase (see text for details and uncertainties); in addition to this early Case B mechanism, some Case A contact binaries may also become unstable and merge as the primary leaves the main sequence. The lower panel shows an example in which a binary initially composed of a $25 M_{\odot}$ star and a $19 M_{\odot}$ star merges to form a $42 M_{\odot}$ single star. That merger product becomes a BSG during core $\mathrm{He}$ burning, then explodes soon after entering the region where LBV outbursts are expected. The dotted part of the curve shows the evolution after the end of core He-burning, although uncertainties in our understanding of such stellar envelopes mean that the precise shape of that part of the curve is very unlikely to be accurate. (This figure has a reduced resolution for astro-ph.)

from a formation channel that is similar to the one which we explore in detail.

As yet another possibility, massive stars in dense young clusters do not need to experience a standard binary instability to be involved in a merger: for example, stellar dynamics can directly lead to collisional mergers. One previous suggestion for the progenitor of SN 2006gy involved the formation of a very massive star by runaway collisions in a dense young star cluster (Portegies Zwart \& van den Heuvel 2007, see also van den Heuvel \& Portegies Zwart 2013). However, this did not explicitly account for the inferred properties of the progenitor 
star at explosion, nor explain why that merger product could produce a strong SN. Our scenario would apply in this case if the multiple mergers occurred soon after the primary had left the main sequence. We will not consider whether the timing of these mergers based on stellar dynamics is likely to occur often enough to be significant. However we will argue that, should early Case B primary stars gain mass from multiple mergers in stellar clusters, this would enable the formation of extremely luminous pre-SN BSGs with cores which still seem likely to avoid direct collapse to a black hole.

\subsection{Aims and structure of this work}

This work examines the proposition that LBV-SN progenitors may form from massive binary systems in which the components merge soon after the more massive star has finished core $\mathrm{H}$ burning, i.e., as it is expanding across the Hertzsprung Gap (HG). As described above, we nominally consider the situation in which the stellar merger follows mass transfer from the early HG primary to the secondary and then a brief period as a contact binary. However, most of the calculations we present would also apply to other situations in which a massive star might gain mass during that evolutionary phase. Therefore this manuscript will describe the stellar models we create as both "post-merger" and "post-accretion".

In $\$ 2$ we study the appearance and evolution of relevant merger products (or post-accretion stars) using Eggleton's stellar evolution code. Partly to demonstrate that our conclusions are robust to reasonable variations in stellar physics we also perform similar calculations using the MESA stellar evolution code, presented in $\$ 3$

In $\$ 4$ we investigate whether these merger products are likely to produce neutron star (NS) or black hole (BH) after core collapse.

Section 5 then estimates event rates for some of the routes through which early Case B accretion might occur, including comparing the potential birth-rates of SN progenitors to current observational constraints. Finally, $\$ 5$ briefly discusses how often one of these early Case B merger products might accrete even more mass from a potential tertiary companion before reaching core collapse.

\section{POST-ACCRETION STELLAR EVOLUTION CALCULATIONS I: USING EGGLETON'S CODE}

\subsection{Assumptions}

We created and evolved a set of post-accretion models using the Eggleton evolutionary code (see, e.g., Eggleton 1971. 1972, 1973; Pols 1994; Pols et al. 1995), and adopting a metallicity of $Z=0.02$. The code assumes the Schwarzschild criterion for convection, and semi-convection is automatically produced by the code's treatment of convective mixing. The equation of state follows the treatment of Eggleton et al. (1973). We adopted the overshooting calibration from Pols et al. (1998), which was performed for intermediate-mass stars but is commonly also assumed for massive-star calculations when using the Eggleton code (see, e.g., Eldridge \& Tout 2004; Eldridge \& Vink 2006). This overshooting is parametrised in a non-standard way, which corresponds to different numbers of pressure-scale-heights for different stellar structures, but the calibrated value is very roughly consistent with 0.25 pressure-scale-heights of overshooting.

Our mass-loss prescription follows the work of Vink et al. (1999). Vink et al. (2000) and Vink et al. (2001) for hot stars.1)

\footnotetext{
1 We adapted the code publicly available from:
}

For stars cooler than $10^{4} \mathrm{~K}$, we adopted a mass-loss rate based on Nieuwenhuijzen \& de Jager (1990), multiplied by a factor of 0.3 to allow for updated estimates of the effect of wind clumping (see, e.g., Puls et al. 2008). In practice, few of the post-accretion models spend much time with surface temperatures cooler than $10^{4} \mathrm{~K}$, and those are the stars in which we are least interested (i.e., they do not end their lives as a BSG).

We first evolved a set of single massive-star models to the $\mathrm{HG}$, and saved snapshots at a range of stages across the HG. To those models we rapidly added mass to their envelopes. These post-accretion models were then evolved to give the sequences shown in Figs. 2 and 3 . To some of the evolved post-accretion models we followed the same procedure again, adding more mass as the star expanded during or after Heburning. One example of such a model is shown in Fig. 2. in Fig. 4 we show that, for the assumptions we have used, the final appearance of stars for which the accretion occurred in one or two phases is relatively small.

We assume that matter is accreted onto the primary star with the surface entropy and composition of the accretor rather than with, e.g., the mean composition and entropy of the secondary star. This assumption deserves further study in future work. However, the thermal structure of the star will recover after a few thermal timescales, i.e., much less than the remaining evolutionary time of the merger product. This suggests to us that our broad conclusions are unlikely to be affected by this assumption, which is supported by the fact that we added mass to primaries with a range of surface temperatures in the HG (and hence studied a range of accretion entropies, as well as slightly different stellar structures at the onset of accretion), but the future evolution of these different post-accretion stars shows only minimal variations, as we will discuss later.

Any systematic bias introduced by not accreting slightly He-rich matter is harder to quantitatively estimate. However, an increase in envelope $\mathrm{He}$ abundance increases the parameter range where stars explode as blue supergiants (Barkat \& Wheeler 1988, 1989; Hillebrandt \& Meyer 1989). Hence slight He-enrichment might be expected to be favourable to the production of BSGs at explosion, and also to delay the point at which the post-accretion star expands to become unstable to LBV outbursts.

As our simulation of the merger process is very simplified, and partly for reasons of numerical stability, we did not include wind mass loss when the merger product is contracting immediately after the merger. The amount of mass lost during this very short time $\left(\lesssim 10^{4} \mathrm{yr}\right)$ is unlikely to be significant, as demonstrated in $\$ 3$, where we perform similar calculations without switching off stellar winds during this phase. We also decided not to apply an ad-hoc LBV mass-loss prescription. Our general conclusions should be unaffected, since the time spent in the LBV phase is extremely short for most of the models presented in this study. Although the final locations of our stellar models in the H-R diagram are not precise, this would have been true whichever LBV mass-loss treatment had been applied.

These calculations include no treatment of stellar rotation. However, since the accretion occurs after strong composition gradients have already been generated by nuclear burning on the main sequence, even rapid rotation caused by accretion of angular momentum is highly unlikely to lead to additional mixing across those gradients (see, e.g., Mestel 1953, 1957. Mestel \& Moss 1986), and therefore we consider that the in- 


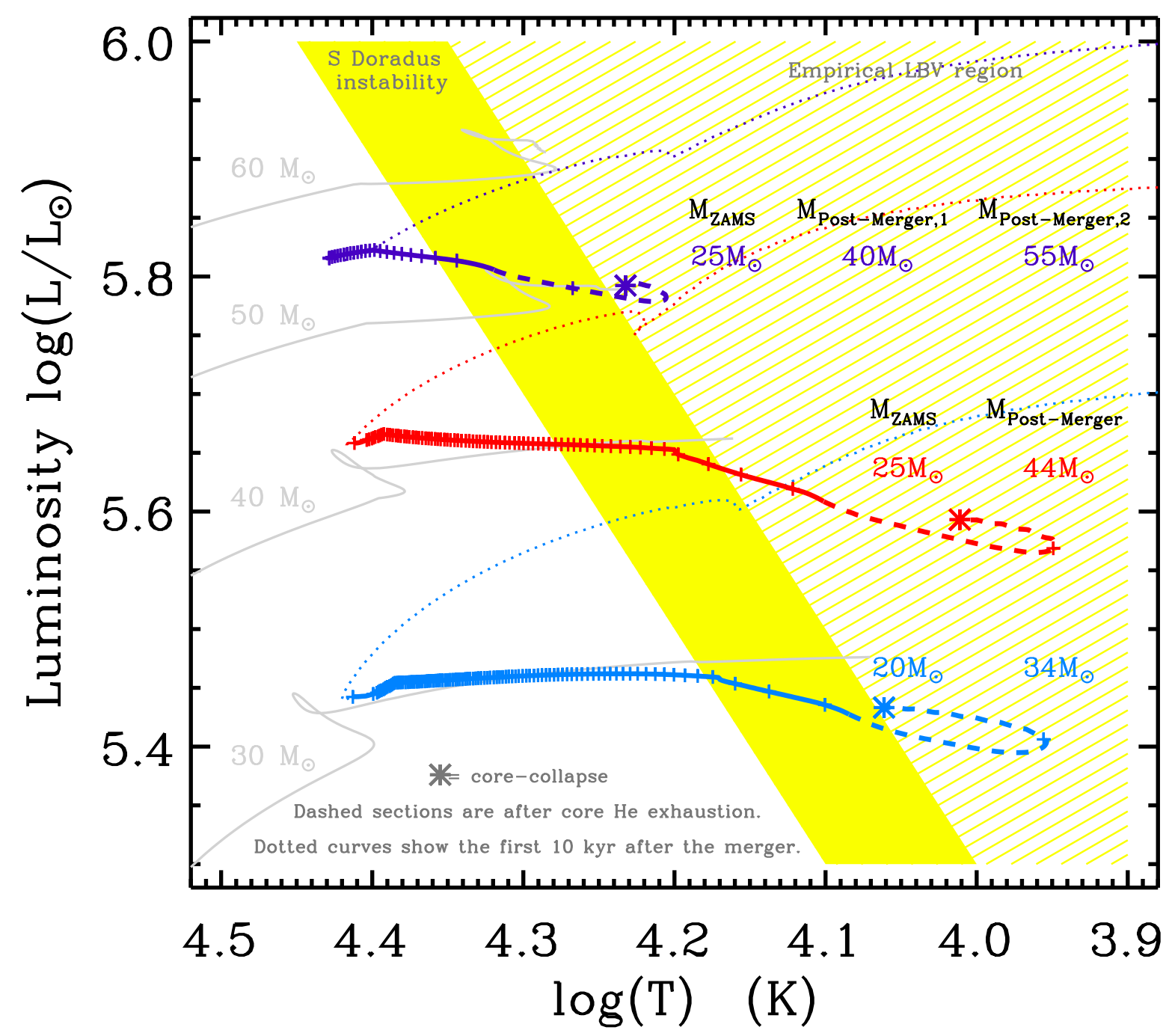

Figure 2. Evolutionary tracks in the Hertzsprung-Russell (H-R) diagram for three post-merger (or post-accretion) stars, represented by the three thicker curves, for which the initial masses $\left(M_{\text {ZAMS }}\right)$ and post-merger masses are as indicated. Of those, the lower two curves represent the evolution of stars that merged soon after the completion of core $\mathrm{H}$ burning (these are blue and red in the online version). Most of the post-merger lifetime is spent undergoing core He burning, shown by the solid section of each curve; the ' + ' signs mark points in the evolution separated by $10^{4}$ years. The dashed section shows the post-core-He-burning phase, whilst the dotted section represents the brief post-merger contraction. The first merger in each case occurs when the primary star had a surface temperature of $10^{4.3} \mathrm{~K}$. The uppermost of the post-merger curves shows an evolutionary track which may be produced by a triple star interaction (this is purple in the online version). In that case, the second merger or accretion event was triggered at a surface temperature of $10^{4.25} \mathrm{~K}$ (see $\$ 5.3$ for a discussion of the likelihood of similar events). The early evolution of four representative massive single stars is shown as thin grey curves. The empirical S Doradus instability strip and the region where these stars are likely to experience LBV outbursts are also indicated. Note that, especially for the blue and purple curves, only a few tens of thousands of years are spent in a region which is typically considered to be potentially unstable to LBV outbursts.

ternal evolution will not be qualitatively altered by rotation. If the cores of the merger products were greatly spun-up during the merger then their future evolution could be affected. This may sometimes occur, but we expect that in the majority of cases the additional angular momentum will be gained by the envelope rather than the core. (Angular momentum transport between the He core and $\mathrm{H}$ envelope was found to be small in the stellar models calculated by Yoon \& Langer (2005), though those examples were for stars in which the core was rotating more rapidly then the envelope, i.e., the reverse of the seemingly-likely case for these stars.) On the other hand, rapid rotation may well increase the likelihood that LBV outbursts occur, and increase the mass-loss rates from the surface (Langer 1997, 1998). There is some suggestion that the angular-momentum loss from less massive merger products during their brief giant phase may be relatively rapid, but the constraints are far from definitive (Eggleton 2010). Our MESA calculations - presented in $\$ 3$ - include comparisons between non-rotating and rotating post-merger models.

\subsection{Results}

As the example sequences in Fig. 2 demonstrate, the postmerger stars spend most of their remaining lives burning He in their cores whilst appearing in the H-R diagram to the left of the regions where LBV outbursts are thought to occur. Only late in their nuclear evolution do they start to expand and become potentially unstable to LBV outbursts. For the LBV instability region marked in Fig. 2. both the $34 M_{\odot}$ and $44 M_{\odot}$ 


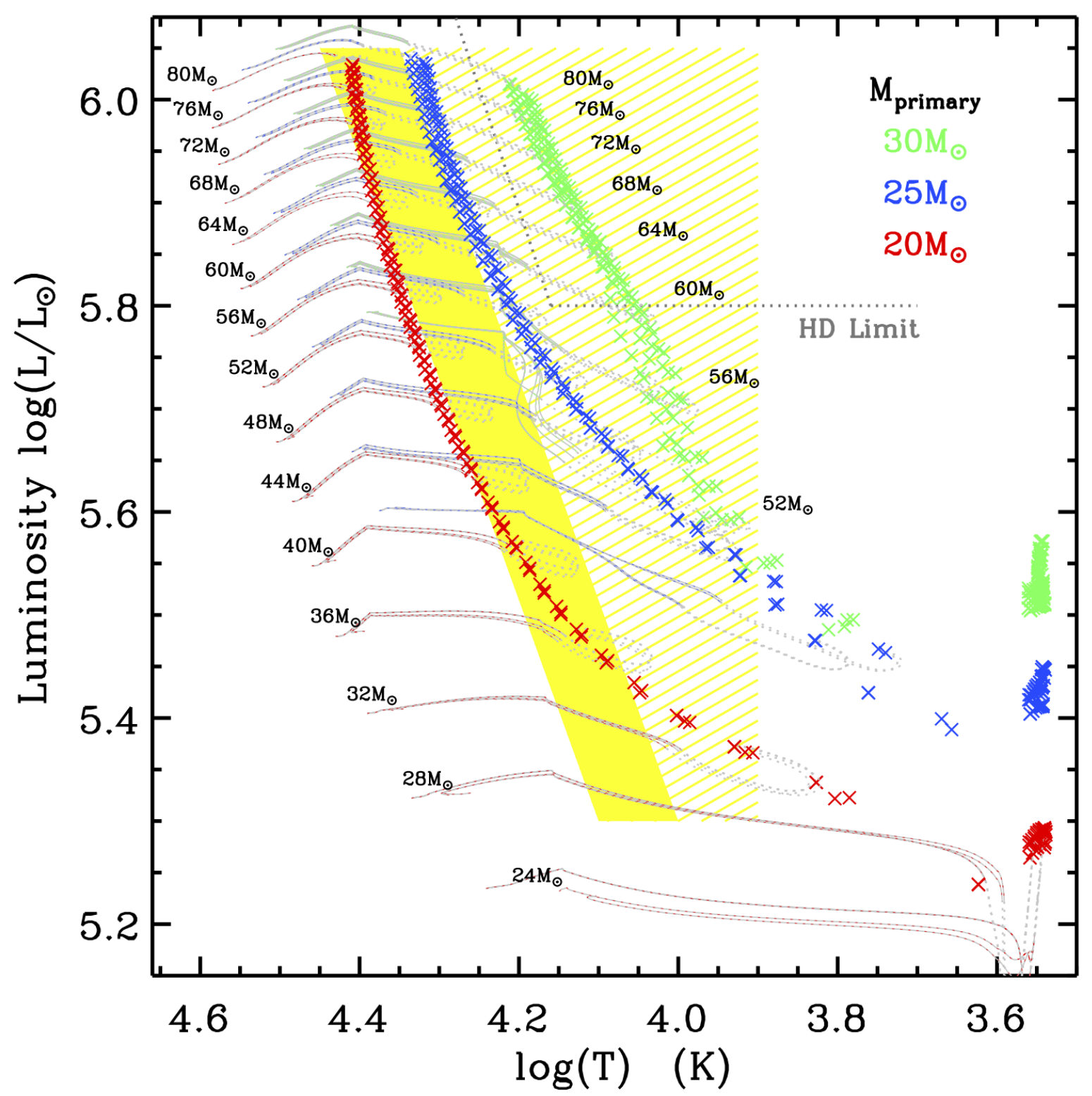

Figure 3. A systematic exploration of the final location in the H-R diagram for a range of stars which gain mass during their Hertzsprung Gap for three initial primary masses $\left(20,25\right.$ and $30 M_{\odot}$, which respectively produce the three sequences from left to right, and which are respectively colored red, blue and green in the online version). Not all of these models represent states accessible to the merger scenario which we consider through most of this work, but the most massive ones indicate what might happen after, e.g., multiple mergers. Models were evolved in increments of $1 M_{\odot}$ in post-accretion mass between the initial primary mass and $80 M_{\odot}$, with the endpoints marked as crosses. For the marked masses, the evolutionary tracks are also shown (the first 10 kyr are omitted, and the dashed segment again represents the post-core-He-burning stage). For each post-merger mass, the different tracks represent different points in the HG at which the merger occurred (see text). Increasing mass gain allows a star to reach core collapse as a yellow supergiant or LBV. (This figure has a reduced resolution for astro-ph.)

examples would only spend their last few tens of thousands of years unstable to outbursts.

One uncertainty which seems to have little qualitative effect on the later evolution is the timing of the accretion (or merger) within the early HG. For the calculations displayed in Fig.3. the ranges of surface temperature at the time of the mergers was $10^{4.3} \mathrm{~K}-10^{3.8} \mathrm{~K}$ (for the $20 M_{\odot}$ and $25 M_{\odot}$ primary stars) and $10^{4.3} \mathrm{~K}-10^{4.0} \mathrm{~K}$ (for the mergers involving a $30 \mathrm{M}_{\odot}$ primary). These temperatures correspond to ranges in mass at the onset of accretion of 19.49-19.45 $M_{\odot}, 23.80-23.45 M_{\odot}$ and 27.86-27.75 $M_{\odot}$ for ZAMS masses of 20, 25 and $30 M_{\odot}$, respectively. Since this change in the timing leads to relatively minor changes in the post-merger evolution, we conclude that the precise timing of the onset of accretion within the early $\mathrm{HG}$ is not significant for our current study. Fig.5 shows that the post-merger lifetime is affected by the timing of the merger, but in most cases the fractional difference is small. The range of difference introduced by changing the timing of the merger could easily be smaller than the other uncertainties in the stellar physics. 


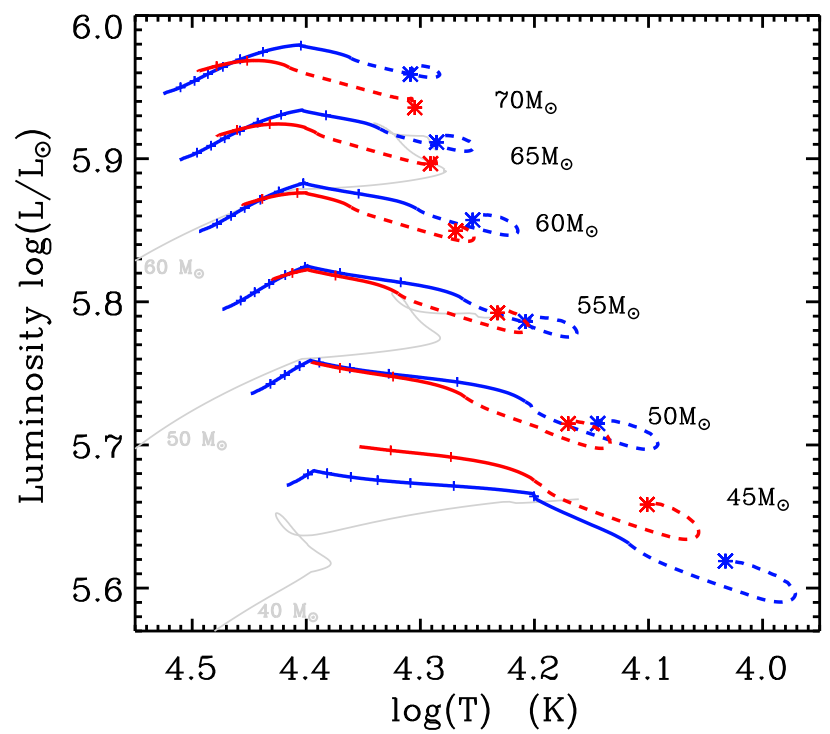

Figure 4. We demonstrate that the final appearance of post-accretion stars are broadly unaffected by whether the accretion occurs in one phase or two, for a representative set of models. As in Fig.2 the dashed segments mark post-core-He-burning evolution, and we show the early evolution of some massive single stars with thin grey curves. Here the ' + ' mark points separated by $10^{5}$ years. As in Fig. 3 we omit the first $10 \mathrm{kyr}$ of the post-accretion evolution. The initial primary mass for all cases was $25 M_{\odot}$. The darker thick curves (blue in the online version) represent models in which there was only one phase of accretion, which began when the primary had a surface temperature of $10^{4.3} \mathrm{~K}$. Further accretion onto the $40 \mathrm{M}_{\odot}$ post-accretion model from that set was used to create the models represented by the lighter thick curves curves (red in the online version). That second phase of accretion began when the first post-merger star had a surface temperature of $10^{4.25} \mathrm{~K}$. We compare pairs of models with the same post-accretion mass (as marked on the plot).

This logarithmic decrease of 0.5 dex in effective temperature corresponds to a radius increase by a factor of 10 (assuming constant luminosity, as is roughly appropriate for the early HG). Our population estimates later adopt a factor of 10 radius increase within the $\mathrm{HG}$ as the range of parameter space during which mergers can lead to suitable SN progenitors. Based on these calculations, we suggest that this factor of 10 in stellar expansion may well be conservative.

Since the location in the HR diagram where LBV outbursts occur is not precisely known - and, as noted above, may well be influenced by rotation - the S Doradus instability strip marked in Figs. 2 and 3 can only be indicative. Groh et al. (2009) argued that the 'minimum LBV instability strip' is steeper in the HR diagram than in previous work, located at an effective temperature of $\approx 10^{4.2} \mathrm{~K}$ for a luminosity of $10^{5.414} L_{\odot}\left(\right.$ and $\approx 10^{4.3} \mathrm{~K}$ for a luminosity of $\left.10^{5.9} L_{\odot}\right){ }^{2}$ This is typically slightly hotter than the left edge of the instability strip marked in Figs. 2 and 3. Figure 6 presents the length of time for which our models stars would be unstable to LBV outbursts for these different assumptions. Note that the plots do not include any arbitrary assumptions about a minimum absolute luminosity for LBV outbursts. Nonetheless from Figs. 3 and 6 it is clear that - wherever the real minimum instability strip lies - models can be created which would only become unstable to LBV outbursts very late in their evolution, potentially even after the end of core He burning. As the instability strip moves to higher temperatures then the primary

2 Groh et al. (2009) give $\log \left(L / L_{\odot}\right)=4.54 \log \left(T_{\text {eff }} / \mathrm{K}\right)-13.61$.

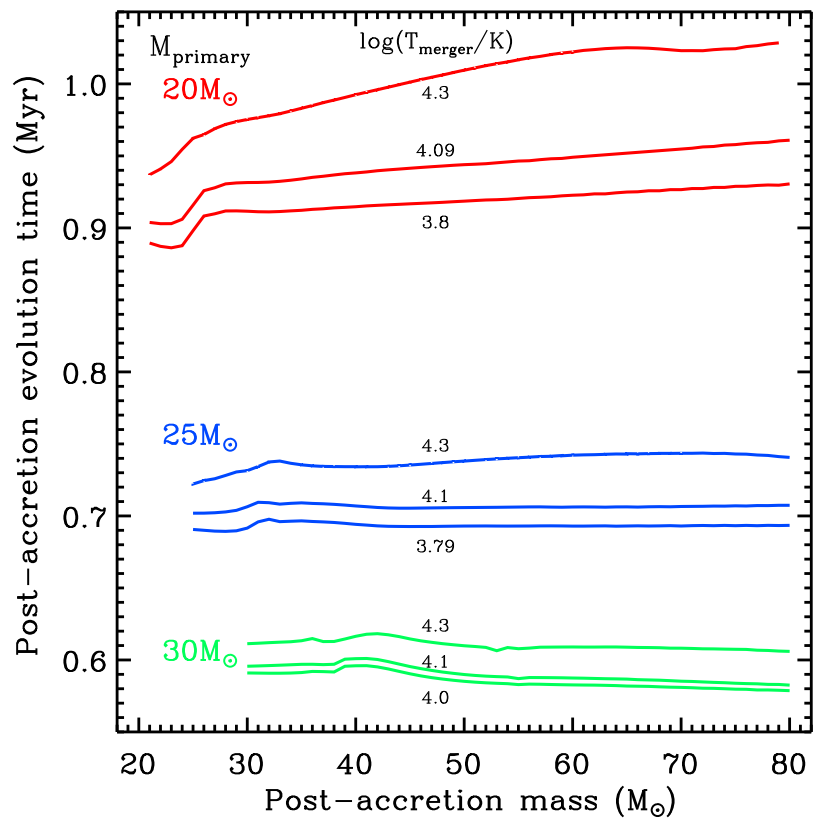

Figure 5. For our set of calculations using Eggleton's code, we show the duration of the post-merger evolution as a function of the post-accretion mass (i.e., nominally until the end of core carbon burning; carbon burning is numerically unstable, and not all models complete that phase successfully, but that would only produce a negligible error in this duration). The lifetimes of the merger products are governed by the the primary mass (as marked), and in most cases are only weakly affected by the amount of mass accreted. The surface temperature of the primary at the start of accretion is marked by each curve; this factor does have some effect on the lifetime of the merger product, with smaller (i.e., hotter) pre-accretion stars living somewhat longer.

mass required to achieve this becomes lower. This systematic effect suggests that as the real instability strip becomes hotter then higher post-merger luminosities will be harder to achieve for canonical binary merger channels (and so would perhaps require, e.g., multiple mergers in dense stellar systems or triple-star scenarios). Cooler instability strips would enable merger products resulting from increasingly massive primaries to become unstable later in their post-accretion evolution.

The criteria adopted in Fig. 6 are very uncertain, and the results shown therein can only be rough estimates. Nonetheless, we note that the time spent subject to LBV outbursts is predicted to be a fairly sensitive function of the post-accretion mass, which is in strong contrast to the post-merger lifetimes shown in Fig. 5 .

\subsection{Yellow supergiants}

Figure 3 also indicates that there is a region of parameter space where merger products finish their nuclear burning as yellow supergiants (YSGs). Such SN progenitors were again not predicted in canonical single-star models but have been suggested observationally (see, e.g., Fraser et al. 2010). The models which lead to core collapse as YSGs are those systems in which less mass was added to the primary than necessary to produce BSG or LBV SN progenitors. For example, the $40 M_{\odot}$ post-merger star produced from a $25 M_{\odot}$ primary finishes its evolution with a surface temperature of $10^{3.8} \mathrm{~K}$.

\section{POST-ACCRETION STELLAR EVOLUTION CALCULATIONS II: USING MESA}



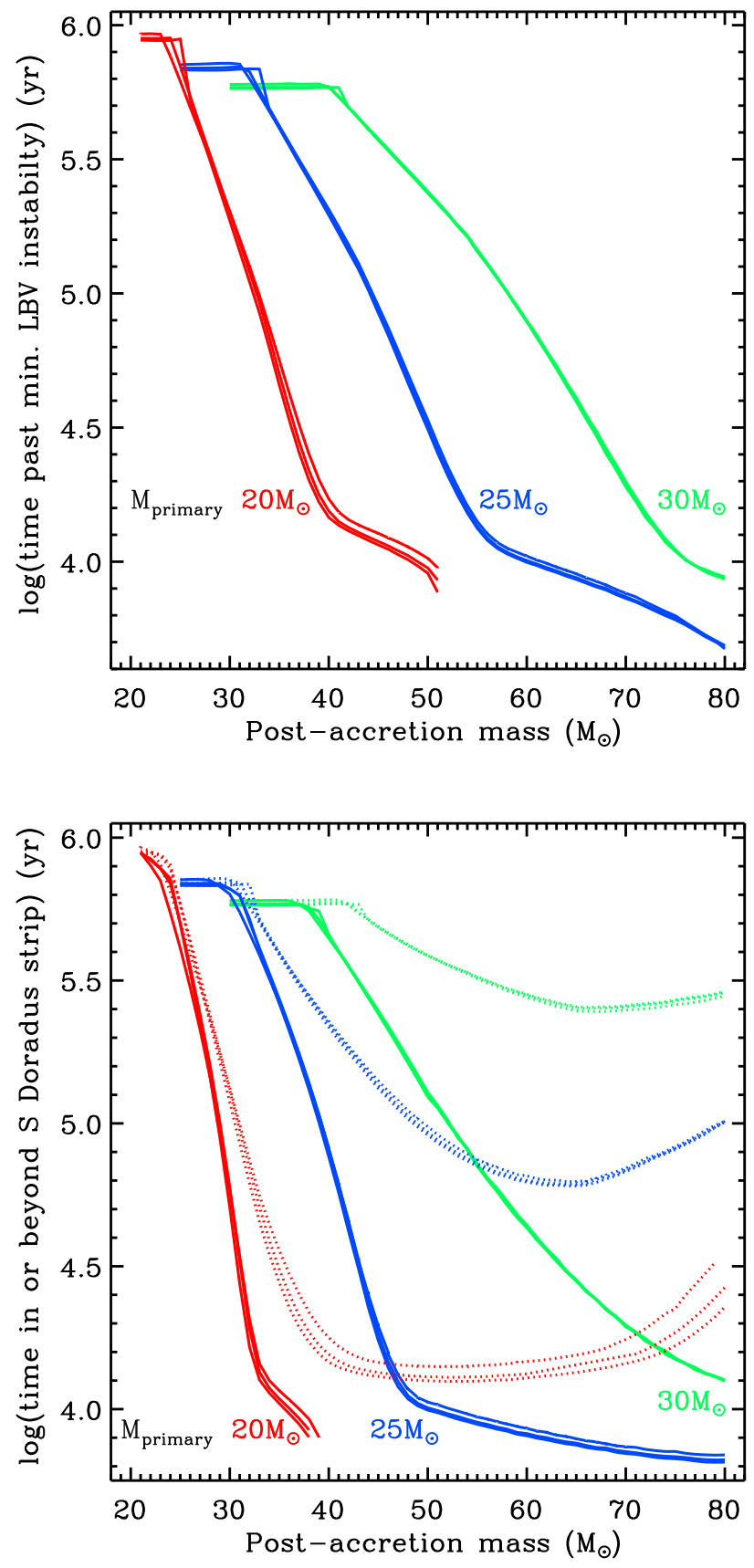

Figure 6. Based on the calculations in $\$ 2$ and different inferred empirical LBV instability regions, we estimate the length of time for which our postmerger models might be subject to LBV outbursts. Again, for each primary mass (as marked) we show results for different timings of the merger within the HG; in this case, this almost always leads to small differences in outcome. The upper plot shows the length of time for which the post-merger models exceed the minimum LBV instability criterion given in Groh et al. (2009). The lower panel uses the S Doradus instability strip plotted in Figs. 2 \& 33 as the instability criterion, with the broken curves using the left edge of the strip, and the solid curves the right edge of the strip. We stress that these are only intended to be rough estimates (especially since the criteria for LBV instability are not well understood), and also that these criteria adopt no minimum luminosity cut-off (i.e., it is extremely unlikely that a $25 M_{\odot}$ post-merger star would display LBV outbursts).
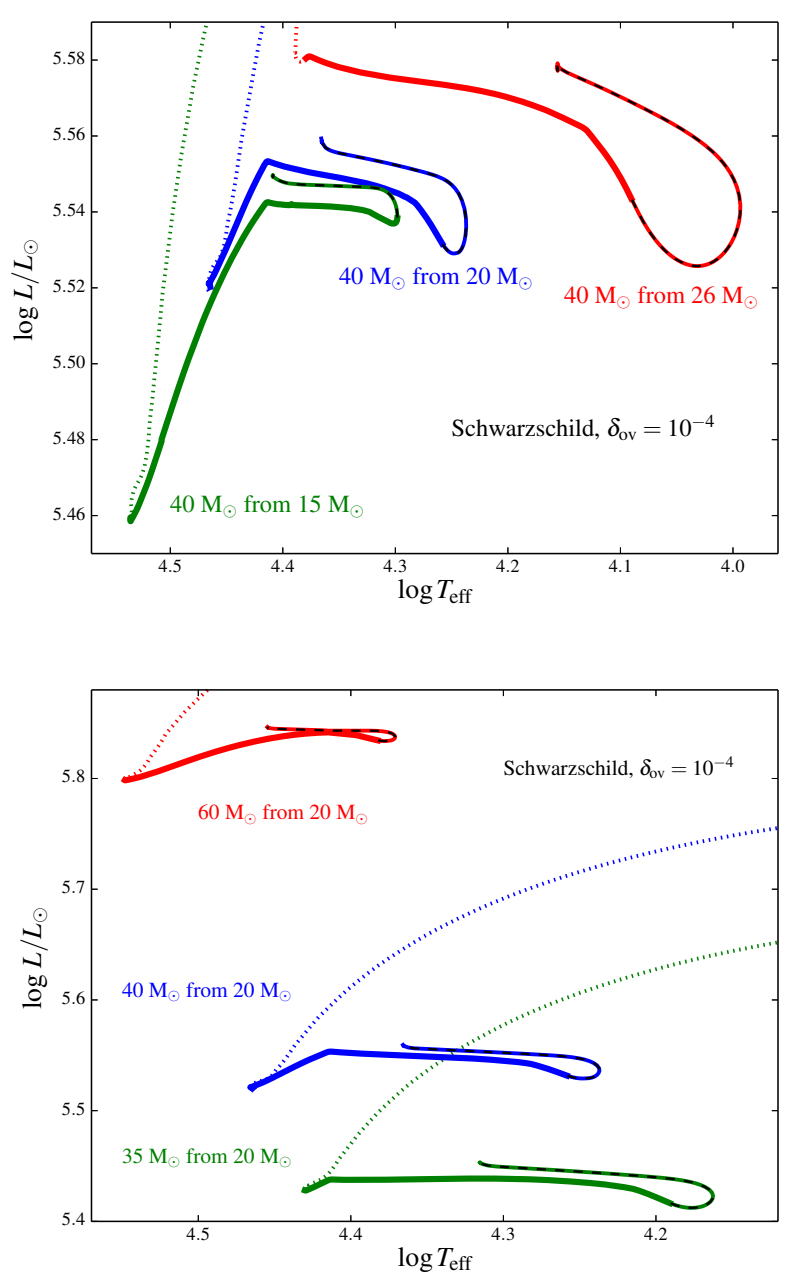

Figure 7. The features of the post-accretion evolution for our MESA calculations are similar to those shown in $\$ 2$ These MESA models are of non-rotating stars and adopt the Schwarzschild criterion with overshooting (see text). The dotted curves represent the post-accretion thermal contraction phase, whilst the black-and-color dashed sections of the curves represent the post-core-He-burning phase. For all of these models, the accretion began when the radius of the primary was $30 R_{\odot}$. Upper panel: we compare the evolution of a $40 M_{\odot}$ star formed from three different primary masses (as marked) by early Case B accretion. Lower panel: we compare the appearance of three stars each formed by early Case B accretion onto a $20 M_{\odot}$ primary, with three different post-accretion masses (as marked).

We have no reason to doubt the calculations presented in $\$ 2$ Nonetheless, we recognise that the evidence in favour of our model would be strengthened by presenting further calculations using an alternative code, especially since models for the BSG progenitor of SN 1987A were dependent on the assumed physics (see, e.g., Weiss 1989; Langer et al. 1989. Podsiadlowski 1992). For this purpose we use MESA (as presented by Paxton et al. 2011, 2013).

The MESA calculations presented in this paper were performed using version 5232. We chose to use the "Dutch" wind-loss option, based on the choices made in Glebbeek et al. (2009), which is similar to the wind-loss rates adopted in $\$ 2$ We again adopted a metallicity of $Z=0.02$. For the calculations presented here we used the streamlined nuclear reaction network "approx 21 ", which allows us to follow approximate nuclear burning to the formation of an iron core.

The majority of the results presented were calculated us- 
ing the Schwarzschild criterion for convective instability. We later compare calculations using the pure Schwarzschild criterion (with no overshooting), and the Schwarzschild criterion with significant overshooting. For overshooting we adopt the standard treatment in MESA, which follows the exponential overshooting treatment of Herwig (2000); the set of calculations with overshooting takes all of those exponential overshooting parameters, $\delta_{\text {ov }}$, to be $10^{-4}$. We will also present a set of calculations which use the Ledoux test for convective instability, in which we adopt very efficient semi-convection $\left(\alpha_{\mathrm{SC}}=0.1\right)$. Uncertainties in stellar mixing physics are still substantial, and we certainly have not tested all possible options. However, since our parameter choices with respect to overshooting and semi-convection are on the larger side of the plausible range, if they are in error, they seem likely to produce cores which are more massive than might be the case in reality. More massive cores (i.e., higher fractional core masses) are less favourable when trying to produce BSG structures. Therefore - for the scenario we are testing in this paper - we consider these parameter choices to be conservative. We expect that less substantial overshooting, or less efficient semi-convection, would be more favourable for the production of massive BSGs.

We adopted a similar procedure and assumptions to produce the merger products as when using the Eggleton code except that when using MESA we did not temporarily switch off wind loss during the brief post-merger contraction. The pre-merger stellar models, and the accretion phase, were typically calculated with higher resolution than the MESA default (setting the mesh-spacing parameter $C=0.1$, and with the maximum allowed number of mesh-points increased to 40,000). Our post-accretion calculations typically adopted the default resolution (with $C=1$ ). For all of the models presented here, the assumed accretion rate during the merger phase was $10^{-2} M_{\odot} \mathrm{yr}^{-1}$.

\subsection{The effect of post-merger rotation}

As noted in $\$ 2$ there is good reason to expect that additional mixing effects due to rotation are minimal across strong molecular-weight gradients (see especially Mestel 1953. 1957; Mestel \& Moss 1986). Since our pre-merger stars already have H-exhausted cores, we therefore considered that the internal evolution of the post-accretion stars is unlikely to be qualitatively altered by rotationally-driven mixing. Here we attempt to test that assumption, by adopting the default set of rotational-mixing physics in the version of MESA used for these calculations. This treatment is based on Heger et al. (2000, 2005), with the main parameter - the ratio of the turbulent viscosity to the diffusion coefficient - set to $1 / 30$. Since rotationally-driven mixing is poorly-understood, this test cannot be considered exhaustive. In addition, we cannot exclude the possibility that a different post-merger angular momentum distribution would have produced qualitatively different results. We simply assumed solid-body rotation at the end of the accretion phase, which seems the most plausible assumption for the deep convective envelopes possessed by the postaccretion stellar models. However, it is possible that stellar cores may be spun-up as a result of mergers.

Despite those caveats, we consider that our results support our assumption that rotational mixing does not make a significant qualitative difference to the evolution of these postmerger stars, at least after the initial contraction phase. Two representative examples are presented in Fig. 8. The only sig-
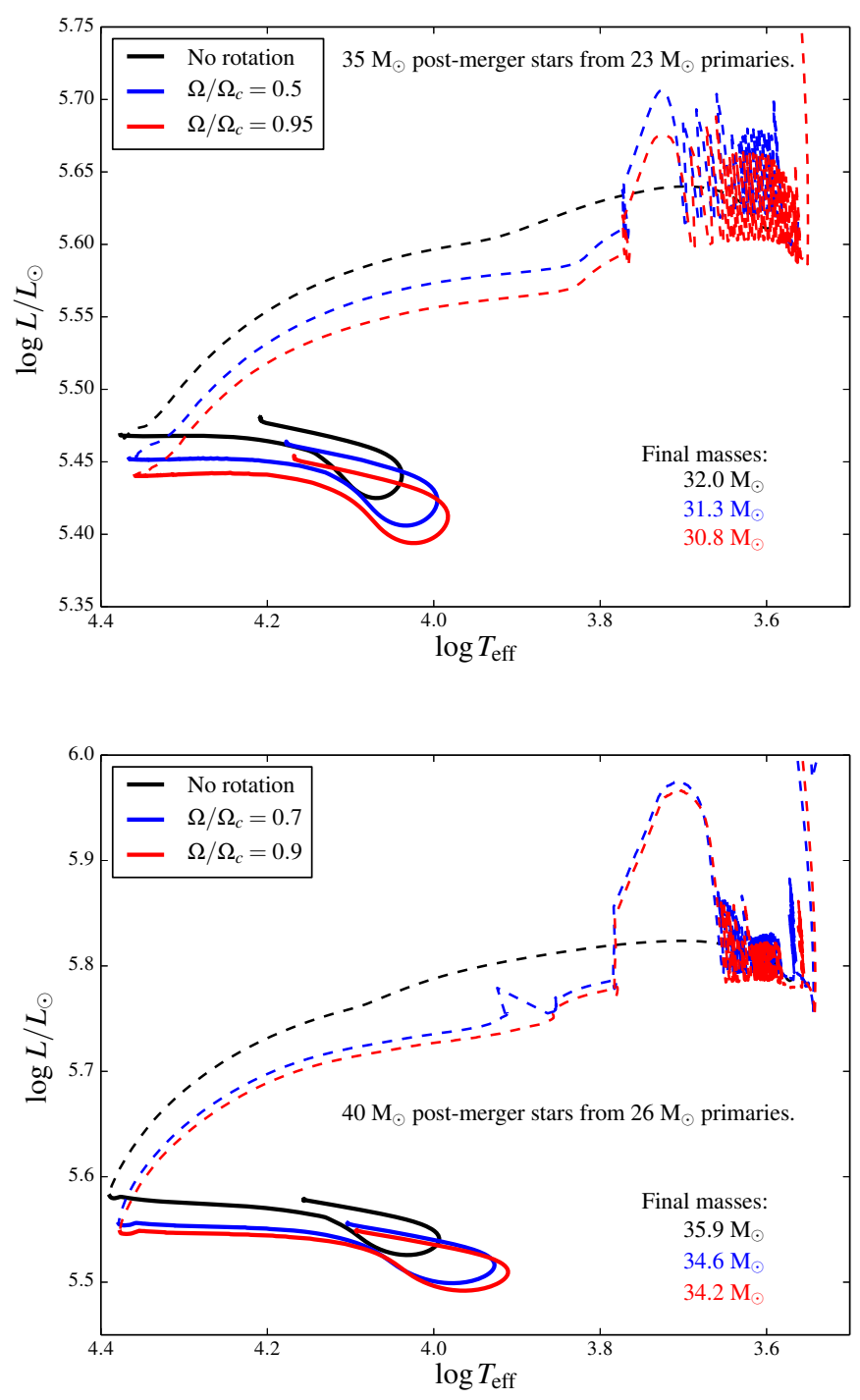

Figure 8. We show the effect of changing the assumed post-merger spin on the stellar appearance for two different merger models $\left(35 M_{\odot}\right.$ from a $23 M_{\odot}$ primary in the upper panel, and $40 M_{\odot}$ from a $26 M_{\odot}$ primary in the lower panel; all models adopt the Schwarzschild criterion for convection with overshooting). The rotation rates are as marked, given as a fraction of the critical rotation rate. As in Fig. 2 broken curves plot the first $10^{4} \mathrm{yr}$ after the merger. Whilst there are differences during the initial contraction phase, which are associated with mass loss and lower final stellar masses, the later qualitative evolution is broadly unaffected by even large rotational velocities.

nificant effect of even extreme post-accretion rotational velocities is mass shedding as the merger product contracts and spins-up (see, e.g., Heger \& Langer 1998). Fig. 8also displays the final masses of the model stars; more rapidly-rotating post-merger models do indeed lose more mass before core collapse.

\subsection{The effect of assumptions about convective mixing}

In Fig. 9, we present one representative example in which the same stellar evolution scenario (both pre-merger and postmerger) was followed using the three different choices of convective instability physics which were described at the start of this section. The final location of the models in the HR diagram is surprisingly similar for all three options, but the shape of the evolutionary track for the model which adopts the 


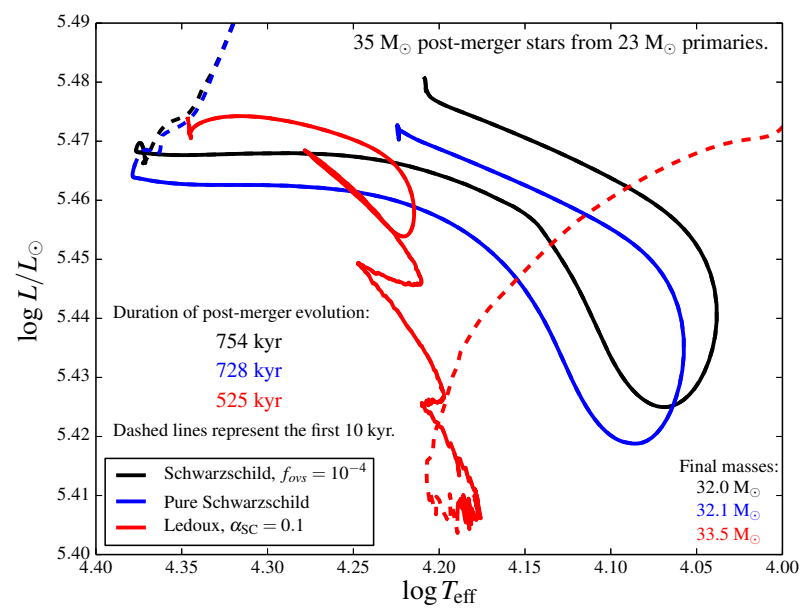

Figure 9. We compare three stellar calculations when adopting different assumptions about mixing (as marked), for the same primary and post-accretion masses $\left(23 M_{\odot}\right.$ and $35 M_{\odot}$, respectively). The post-merger lifetime and final stellar masses are marked on the plot. The post-accretion evolution of the models which assume the Schwarzschild criterion is qualitatively similar, despite the difference in overshooting. However, adopting the Ledoux criterion does lead to a qualitatively different evolution in the HR diagram; most notable is the appearance of sharp turning points which are characteristic of breathing pulses during core He burning.

Ledoux criterion for convection is qualitatively very different from those which adopt the Schwarzschild criterion. It is well known that the shape of blue loops is sensitive to which mixing criterion is chosen, and we speculate that the difference in the shape of the tracks may be a combination of that effect with breathing pulses in the core during He burning. The post-merger lifetime of the Ledoux model is also significantly shorter than for the other calculations, meaning that the star loses less mass before reaching core collapse.

Despite those differences, the basic result that early Case $\mathrm{B}$ mergers can lead to BSGs at explosion is robust against changing the mixing physics in these ways.

\section{THE FATE OF THE CORE: BLACK-HOLE VERSUS NEUTRON STAR PRODUCTION}

In this section we investigate the outcome of core collapse for our models for LBV progenitors of luminous SNe. The particular phase of accretion we have considered - and the merger scenario through which that accretion may occur enables stars to exist which are not only BSGs immediately before core collapse but are also plausibly luminous enough to be potential LBVs. However, if those post-accretion stars were not to produce SNe with standard (or greater) explosion energy, then this model would still fail to explain the observed SNe which have been inferred to have LBV progenitors.

It is widely expected that the core-collapse SN engine somehow uses the gravitational potential energy which is liberated during core collapse to power the SN explosion (see, e.g., Colgate \& White 1966) However, theorists have so far been unable to definitively and robustly model the operation of that mechanism (see, e.g., Woosley \& Weaver 1986; Herant et al. 1994, Fryer 1999, Janka et al. 2007, Ugliano et al. 2012; Müller et al.|2012; Bruenn et al.|2013; Hanke et al. 2013. Fryer 2013, Couch \& O’Connor 2014;, Takiwaki et al. 2014; Fernández et al. 2014). Hence we cannot accurately predict the outcome of core collapse for our stellar models. Nonetheless, the qualitative expectation is that the more tightly bound

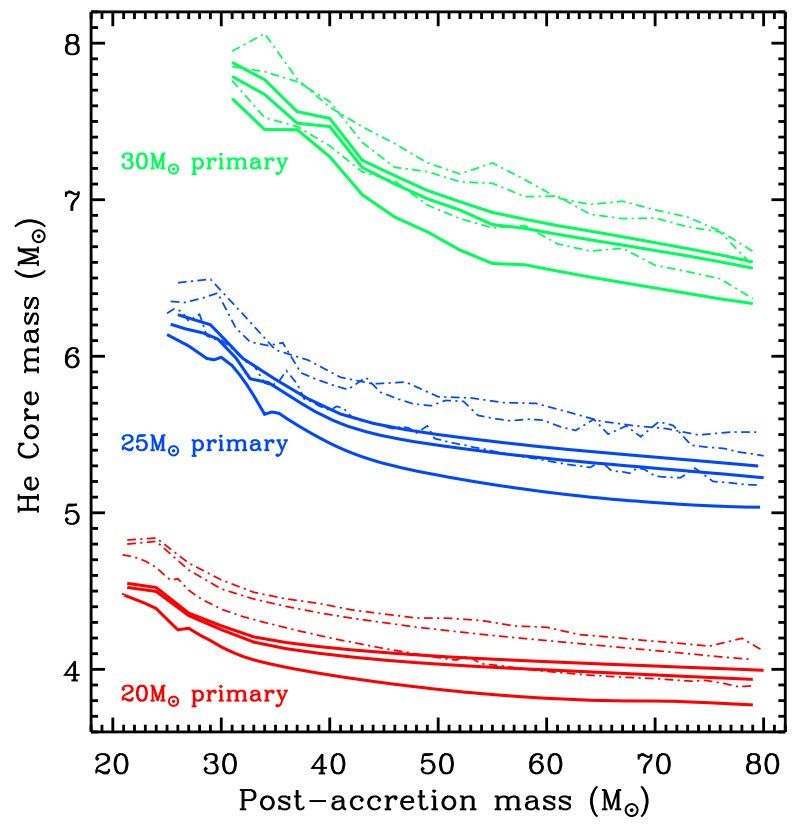

Figure 10. We show the mass of the He core - where the boundary of the core is defined to be where the $\mathrm{H}$ mass fraction is lower than 0.01 - for the post-accretion calculations described in $\$ 2$ The solid curves show the core masses at the point when He core-burning ends, whilst the broken curves are at the end of the calculation (typically after core carbon burning is complete, which can sometimes introduce numerical instabilities). The different models displayed for each primary mass are for different merger times, as in Figs. 3 . 5 \& 6 Early Case B accretion decreases the He core masses at the end of core He burning, with more accretion leading to less massive He cores.

stellar cores of more massive stars are those in which the SN shock stalls and fails to escape, leading to $\mathrm{BH}$ formation and a faint SN (or simple direct collapse with no explosion; see, e.g., Fryer 1999, Heger et al. 2003). In this respect there are commonly thought to be three regimes for the outcome of iron-core collapse:

- formation of a NS;

- formation of a BH 'by fallback' (a NS transiently forms, but the outward-moving shock is not strong enough to unbind the remainder of the core, and this core material is accreted by the NS, which then collapses to a $\mathrm{BH})$;

- formation of a BH by direct collapse.

NSs might also form from "electron capture" SNe, i.e., not from such iron core collapse (see, e.g., Podsiadlowski et al. 2004), which may well also produce faint SNe.

It is uncertain how luminous a $\mathrm{SN}$ should be expected to be associated with the formation of a black hole by fallback, although expectations and observations tend to favour faint fallback SNe (see, e.g., Moriya et al. 2010b). Despite this, support for strong shocks from fallback SNe would follow if SN 1987A were demonstrated to have formed a low-mass $\mathrm{BH}$, as has occasionally been argued (Brown \& Bethe 1994; Brown \& Lee 2004).

Perhaps more robustly, expectations exist for the outcome of core collapse for single stars (or stars which are effectively single) which are mainly based on inferences from observations. Hence we will compare the core properties of our binary models to the core properties of single-star models. We 
will assume that the $\mathrm{H}$-rich envelopes are broadly irrelevant to the question of whether a successful SN shock develops and escapes the star, except for the influence that they have on the structure of the core 3

The expectation for the upper end of the single-star zeroage main sequence mass which produces a NS is sometimes quoted as being between 20 and $25 M_{\odot}$ (see, e.g., Heger et al. 2003, Eldridge \& Tout 2004). This is broadly consistent with the observationally-inferred upper limit on the progenitor mass for type IIP SNe, although there is some tension between the data and simple predictions, with an upper-limit for directly-inferred progenitor masses which is somewhat below $20 M_{\odot}$ (Smartt 2009). However, the observational evidence from type IIP SNe may be compatible with luminous $\mathrm{SNe}$ being produced by ZAMS stars slightly more massive than $20 M_{\odot}$ for reasonable expectations about dust formation obscuring those more massive SN progenitors (see, e.g., Walmswell \& Eldridge 2012).

It is trivial to allow an initially much more massive star to produce a NS remnant, simply by early removal of the star's $\mathrm{H}$ envelope (Belczynski \& Taam 2008). However, the SNe which we seek to explain are H-rich (as are LBVs); therefore this mechanism cannot be directly relevant to these events.

Some previous work has suggested that the properties of the $\mathrm{CO}$ core set the properties of the final iron core which, in turn, controls the outcome of core collapse (Timmes et al. 1996. Woosley \& Timmes 1996, Brown et al. 2000, 2001). However, both Fryer et al. (2002) and Sukhbold \& Woosley (2014) stress that the fate of the core is not a monotonic function of $\mathrm{CO}$ core mass, even if the $\mathrm{CO}$ core mass is broadly a useful indicator of the likely outcome. There are some significant uncertainties in those results and in our calculations, especially arising from the the nuclear reaction cross section for $\alpha$-capture onto ${ }^{12} \mathrm{C}$ (see, e.g., Woosley \& Weaver 1986 Brown et al. 2001; Brown \& Lee 2004), as well as both physical and numerical issues with the treatment of convection (see, e.g., Sukhbold \& Woosley 2014 and references therein, especially Rauscher et al.2002). We also note that rapid rotation may well also affect the outcome of core collapse, even in cases when a "collapsar" does not occur (see, e.g., Fryer \& Heger 2000). The cores of our post-accretion stars are not necessarily rapidly rotating, but this potential effect should also be borne in mind. We will compare the properties of our stellar merger products to those of single-star models calculated using exactly the same code, and only draw conclusions from relative properties rather than absolute values, but these uncertainties could potentially still be problematic.

In what follows we compare a range of indicators for the fate of the core. We will argue that those indicators overall suggest that, despite their increased mass, the post-accretion objects are no more likely to collapse to a $\mathrm{BH}$ instead of a neutron star (NS) than the original primary stars, even if the merger product is massive enough that a $\mathrm{BH}$ remnant would normally have been expected. Whilst the evolution of the post-main-sequence core has not been completely decoupled from the mass of the merger product, the pre-merger mass of the primary seems to be much more important to the final structure of the core than the mass of the merger product. Moreover, and surprisingly, several of the indicators suggest that accretion increases the chance that a star can avoid $\mathrm{BH}$ formation, and thereby increases the range of initial stellar

\footnotetext{
${ }^{3}$ Clearly the dynamical timescale of the $\mathrm{H}$ envelopes are far longer than the core-collapse timescale.
}

masses which produce luminous core-collapse SNe.

\subsection{He core masses from the Eggleton-code calculations}

The Eggleton code is unable to follow stellar evolution all the way to the formation of the iron core. In principle we can calculate the evolution to the end of carbon burning, but numerical instabilities during carbon burning can be troublesome.

Nonetheless, we will compare the masses of the He cores after the end of central He burning as a first indication of the eventual fate of the stellar core. The He core masses are shown in Fig. 10, evaluated both after core He exhaustion and in the final saved model time-step (i.e., nominally at the end of C-burning). Early Case B accretion leads to a decrease in the He core masses. The effect is clearer for 25 and $30 M_{\odot}$ primaries than for $20 M_{\odot}$ primaries, and does depend somewhat on the timing of the onset of accretion (i.e., the temperature or radius of the primary at the onset of accretion) but seems qualitatively generic. A simplistic interpretation of this would be that early Case B accretion may increase the range of initial stellar masses which might produce successful SN explosions at core collapse.

Since Fig. 5 does not show a strong decrease in post-merger lifetimes as more mass is added, the decrease in He core mass shown in Fig. 10 does not appear to be a simple lifetime effect. The effect is at least partly due to dredge-up from the He core by the convective envelope of the post-merger star. This dredge-up may indicate that the details of the thermal relaxation phase after the merger are important for the final fate of the star, in which case our assumptions about the entropy of the accreted material should be examined in more detail. If so, then this uncertainty is related to the possibility that the merger process itself would directly cause dredgeup from the core of the primary (see, e.g., Ivanova \& Podsiadlowski 2003), and to the likelihood that the accreted material will itself be He-enriched (because the the secondary star should have completed some nuclear burning by the time of the merger). As noted earlier, additional He enrichment should be favourable for the production of BSGs (see, e.g., Barkat \& Wheeler 1988, Hillebrandt \& Meyer 1989). Additional dredge-up from the core may also further increase the range of initial stellar masses which can produce a successful core-collapse SN after merger; conversely, it is possible that He-enriched envelopes would sufficiently alter the $\mathrm{H}$ shellburning during the post-merger BSG phase to produce the opposite effect. These combined uncertainties introduced by the physics of the merger/accretion process deserve further study, but they add several dimensions to the parameter space, and so we defer them to future studies.

\subsection{Indications from the post-Si-burning core based on MESA calculations}

MESA enables us to continue calculating the evolution of our models until after the end of core silicon burning ${ }^{4}$ Some of the calculations we present were stopped at the end of central silicon burning, whilst for others we followed the collapse of the core until it reached an infall velocity of $10^{8} \mathrm{~cm} \mathrm{~s}^{-1}$; for results where the difference is important, we will state which termination criterion was used.

\subsubsection{Core profiles of example models}

\footnotetext{
${ }^{4}$ However, we understand that MESA has not been as well validated for silicon burning as for earlier phases (L. Bildsten [priv. comm.]).
} 

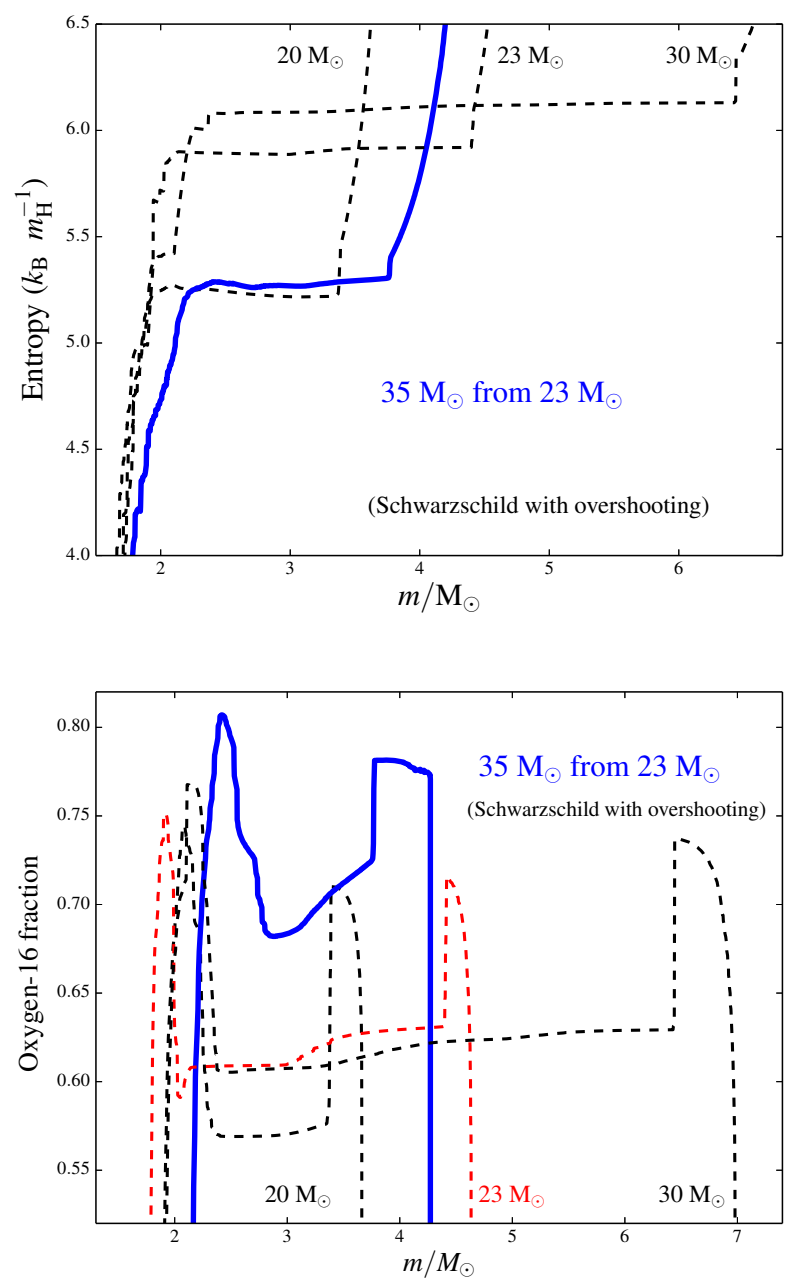

Figure 11. We compare the details of the structure at core collapse for one of our merger products/post-accretion stars with that of three single stars $(20,23$ and $30 M_{\odot}$, as marked). In all cases, these models are shown when the core infall velocity reaches $10^{8} \mathrm{~cm} \mathrm{~s}^{-1}$. The $35 M_{\odot}$ merger product was formed by accretion onto a $23 M_{\odot}$ primary which had a radius of $100 R \odot$ at the onset of accretion and the structure is shown using thick solid curves (blue in the online version). Differences between the merger product and the $23 M_{\odot}$ single star are therefore a result of how the accretion has affected the evolution of the star. The upper panel shows a portion of the entropy profiles of the stellar cores, and the lower panel the ${ }^{16} \mathrm{O}$ composition profile. The entropy profile within the $\mathrm{CO}$ core of the merger product has values closer to those of the $20 M_{\odot}$ single star than those of the $23 M_{\odot}$ model. The mass gained by the merger product leads to a reduced overall mass for the final O-rich core, and the inner boundary of the O-rich material also moves outwards in mass coordinate.

Figures 11 and 12 compare the final core structures of example post-accretion models to similar-mass single-star models. In both of these examples, the final mass of the O-rich core is smaller for the post-accretion star than for the single star with the same initial mass. However, we also note that the shape of the composition profile is not intermediate between any of these single-star model (e.g., the peak mass fraction of ${ }^{16} \mathrm{O}$ is higher than for any of these single stars).

The entropy profiles of the post-merger stars are also altered in the broad direction of lower-mass progenitors, in particular the value of the entropy plateau in the O-rich core moves to lower values ${ }^{5}$ However, the entropy profiles do not change in

\footnotetext{
${ }^{5}$ We note that the fact that some of the entropy profiles slightly decrease
}
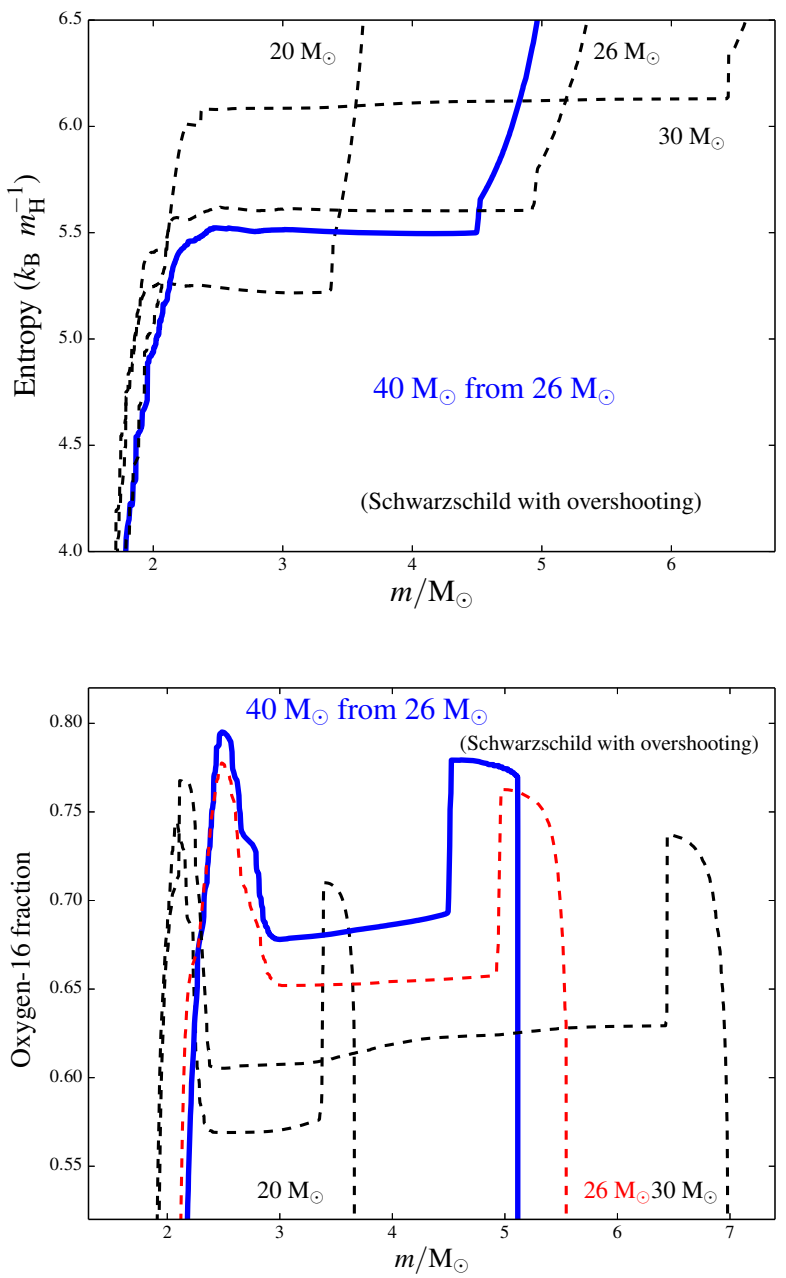

Figure 12. As in Fig.11 we compare details of the entropy and composition profiles from one post-accretion star to those of three single stars. Again, these structures are when the core infall velocity reaches $10^{8} \mathrm{~cm} \mathrm{~s}^{-1}$. In this case, the merger product was a $40 M_{\odot}$ star formed by accretion onto a $26 M_{\odot}$ primary with a radius of $100 R_{\odot}$. Here we also show the structure of a $26 M_{\odot}$ single star for comparison. The qualitative trends are similar to those in Fig. 11 in that both the final $\mathrm{CO}$ core mass and the specific entropy within the $\mathrm{CO}$ core are lower, and the peak oxygen abundance is higher. However, the effects in this case are less drastic. In contrast to Fig.11 the location of the inner boundary of the O-rich material in the merger product is only marginally further out than the boundary in the corresponding single star (i.e., in this case $\left.26 M_{\odot}\right)$.

a simple monotonic way as the single-star mass is increased, since the value at which the $23 M_{\odot}$ entropy profile plateaus is somewhat above the value at which the $26 M_{\odot}$ star does so. This may be related to the suggestion that the cores of stars in the region around $23 M_{\odot}$ may be more difficult to explode than those of stars in the region around $26 M_{\odot}$ (Ilka Petermann, [priv. comm.]; see also Sukhbold \& Woosley 2014). If it is true that stars in the range $\approx 25-30 M_{\odot}$ are relatively "explodable", as concluded by Sukhbold \& Woosley (2014), then this region of initial primary-star masses might be the most favourable for producing LBV SN progenitors via this binaryaccretion scenario. Further examples of how the entropy profile is affected by early Case B accretion are given in Fig.13. which shows how the core entropy profile of a $20 M_{\odot}$ primary 


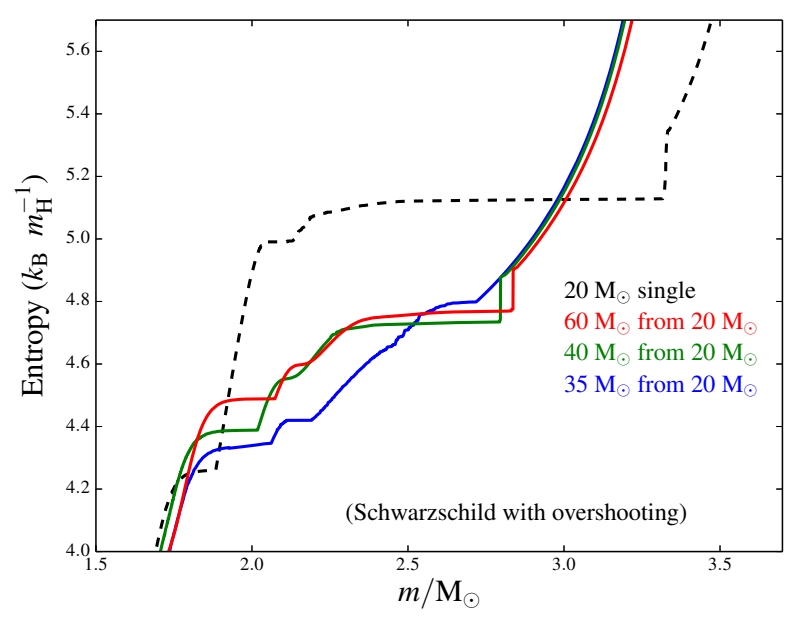

Figure 13. We compare details of the entropy profiles at the end of core Si burning for a $20 M_{\odot}$ single star (the black dashed curve) and three stars formed by early Case B accretion onto such a $20 M_{\odot}$ primary (the solid curves, colored as their respective labels; in all cases the accretion began when the primary star had a radius of $30 R_{\odot}$ ). The entropy profiles in this region of the post-accretion stars are more similar to each other than to that of the single star. Even the $60 M_{\odot}$ post-accretion star produces a less-massive and lower-entropy core than the $20 M_{\odot}$ single star. We also note that these merger products have far less substantial plateaus in their entropy profiles, with the corollary that the entropy gradients in this part of their core are typically shallower; in particular, the $35 M_{\odot}$ post-accretion star finishes $\mathrm{Si}$ burning with no sharp jump in this region of the entropy profile.

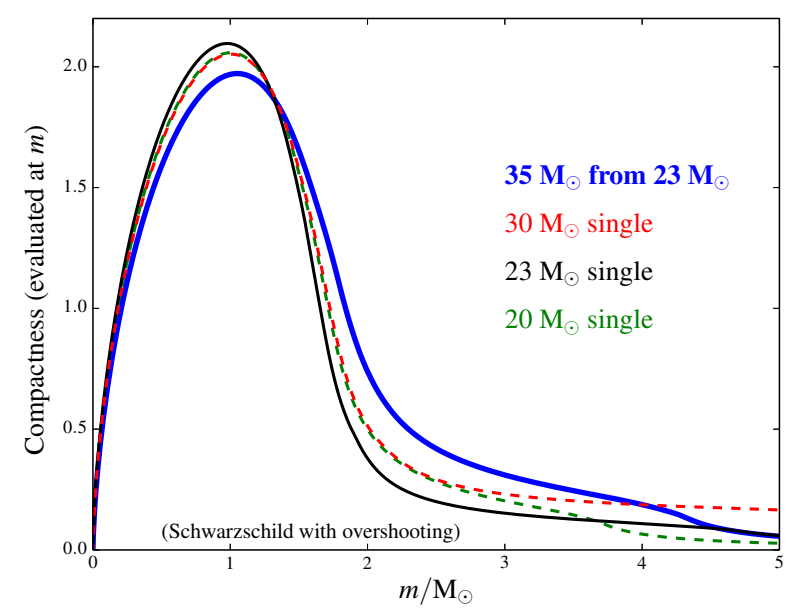

Figure 14. At a core infall velocity of $10^{8} \mathrm{~cm} \mathrm{~s}^{-1}$, we compare the internal profile of the compactness parameter, $\xi$ for four stellar models: three single stars with initial masses of $20 M_{\odot}$ (green broken curve), $23 M_{\odot}$ (black curve) and $30 M_{\odot}$ (red broken curve), and a merger product with a $35 M_{\odot}$ postmerger mass from a $23 M_{\odot}$ primary (the thick blue curve), i.e., the same models as in Fig. 11 A color version of this Figure is available in the online version.

is altered by increasing amounts of mass gain. The specific entropy in this region of the core for post-accretion masses of 35, 40 and $60 M_{\odot}$ are more similar to each other than to that of the $20 M_{\odot}$ single star. Moreover, the entropy profile of the $35 M_{\odot}$ post-merger star in Fig. 13 is distinguished by having almost no plateau in this region, and no sharp entropy jumps. If such changes in the shape of the entropy profile affect how easily these cores explode, then this effect of binary evolution may be very important. We note that for this set of models the change in the shape of the entropy profile is greatest for the lowest amount of accreted mass. We stress that we have not modelled the full parameter space for which early Case B accretion may be important; we suspect that this effect may be import for primary stars which are less massive than those which we have modelled for this work on LBV SN progenitors.

Based on Figs.11, 12 and 13 we might anecdotally conclude that this supports the idea that early Case B merger products and post-accretion stars should be easier to explode than single stars with the same initial primary-star mass, as suggested by the results in $\$ 4.1$ However, an alternative indicator - the compactness parameter $(\xi$, as suggested by O'Connor \& Ott 2011, Ugliano et al. 2012; O'Connor \& Ott 2013) - is less favourable. We do not evaluate $\xi$ at core bounce, as was done by O'Connor \& Ott (2011, 2013). However, Sukhbold \& Woosley (2014) show that conclusions drawn from evaluating $\xi$ at core infall velocities of $10^{8} \mathrm{~cm} \mathrm{~s}^{-1}$ are roughly equivalent to those at core bounce; they further demonstrate that comparing $\xi$ from models at earlier evolutionary phases - even as far back as $\mathrm{O}$ ignition - produces similar, though less well-developed trends to those shown by $\xi$ at core-collapse. The distribution of $\xi(m)$ is shown in Fig. 14 for the same models as shown in Fig. 11. Higher values of this compactness parameter are thought to indicate that cores are more difficult to explode. To some extent, conclusions based on the comparative compactness of these models are sensitive to the location at which $\xi$ is evaluated. The inner core of the merger product is less compact than any of the single stars, but between $\approx 1.5$ and $4 M_{\odot}$, the merger product possesses a higher value of $\xi$ than any of the single stars. (Note also that, over the same range, the $23 M_{\odot}$ single star is less compact than either of the $20 M_{\odot}$ or $30 M_{\odot}$ models). Outside the O-rich layer (for which, see Fig. 11), the compactness of the merger product falls relatively sharply. If the relevant mass scale is smaller than $4 M_{\odot}$, then simple application of $\xi$ suggests that this merger product would be harder to explode than any of the single stars to which we compare it.

\subsubsection{Collected indicators for sets of models}

Figs. 15, 16 and 17 present potential indicators of the outcome of core collapse for collections of single and postaccretion stellar models. There we show four types of characteristics of the cores of those stars:

- The mass of the final O-rich core (as shown for individual cases in Figs. 11 \& 12.

- The binding energy of the outer core. This is shown for both the O-rich layer alone and for all mass from the outside of the Si-rich core to the outside of the Herich core. More precisely, we add the magnitude of the infall kinetic energy to the magnitude of the binding energy. (If proto-NS formation in the inner core releases a roughly fixed amount of energy, and the explosion mechanism also always converts a fixed fraction of that energy release, then this quantity would control whether the SN engine is energetically capable of ejecting the outer core.)

- The compactness parameter evaluated at a mass coordinate of $2.5 M_{\odot}$ (i.e., $\xi_{2.5}$; see O'Connor \& Ott 2011 2013, Sukhbold \& Woosley 2014). 

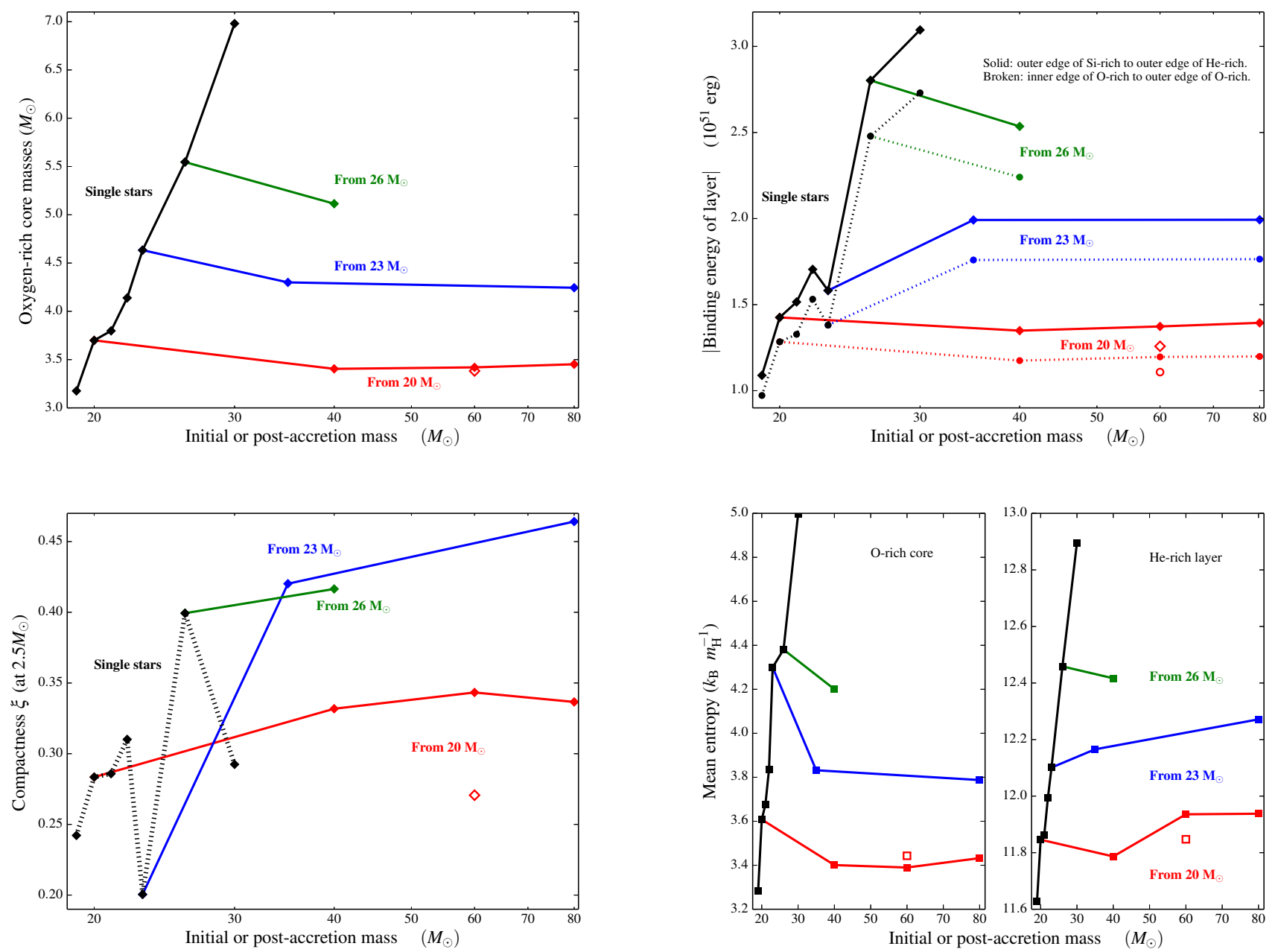

Figure 15. We compare several quantities evaluated at a core infall velocity of $10^{8} \mathrm{~cm} \mathrm{~s}^{-1}$ for single-star stellar models (shown in black) and post-accretion stellar models (plotted using colored lines and symbols). These calculations assumed no stellar rotation and adopted the Schwarzschild criterion for convection with overshooting (as described in the text). For single-star models the abscissa shows the mass of the initial pre-main-sequence model, whilst for post-accretion models the abscissa gives the post-accretion mass (and the initial mass is as labelled). For accretion onto $20 M_{\odot}$ primaries, the solid symbols represent models which began accretion at $30 R_{\odot}$; the larger, hollow symbol represents a case where the accretion began at a primary radius of $100 R_{\odot}$; for the models which represent the outcome of accretion onto $23 M_{\odot}$ and $26 M_{\odot}$ primaries, accretion began at $100 R_{\odot}$. Upper-left: The mass inside the boundary of the O-rich core. Upper-right: The binding energy of the outer core, from the outer edge of the Si-rich core to the outer edge of the He-rich core (shown with symbols joined by solid lines). This binding energy is dominated by that of the O-rich layer (given using symbols joined by broken lines). Lower-left: The compactness parameter, $\xi_{2.5} \mathrm{of}$ O'Connor \& Ott (2013). Lower-right: The mean entropy per baryon within the O-rich core (left) or the He-rich layer outside the O-rich core (right).

- The mean specific entropy inside both the O-rich core and the remainder of He core (i.e., the He-rich layer outside the oxygen core).

For most of the quantities shown in Figs. 15, 16 and 17, either the change in the stellar structure after early Case B accretion is normally weak or the trend resulting from accretion is in the opposite direction to the trend produced by increasing single-star mass. The main exception is the compactness parameter $\left(\xi_{2.5}\right)$, and only for the models which assume overshooting (Figs. 15 and 16. For those models, if $\xi_{2.5}$ were a reliable parameter for predicting black-hole formation at core collapse, then early Case B accretion would broadly increase the likelihood of black-hole formation (since for those stellar models we find that $\xi_{2.5}$ increases with accretion). However, we find that the trend is mostly reversed for the set of calculations without overshooting, for which see Fig. 17 In that case we find that most of the post-accretion models show a lower value of $\xi_{2.5}$ than if the primary had been allowed to evolve without accretion; the $50 M_{\odot}$ star formed from early Case B accretion onto a $23 M_{\odot}$ primary is a strange and strong exception. We also note that $\xi_{2.5}$ shows an unclear trend for the single-star sequence, whether or not we adopt any overshooting when calculating the single stars; this non-monotonic behaviour has recently been studied in detail by Sukhbold \& Woosley (2014).

These comparisons also suggest that $\xi_{2.5}$ may be the indicator for which changing the radius at the start of the accretion phase makes the largest relative difference (see especially Fig. 15, but also Fig. 17), although we have too few direct comparisons to be sure that this is generally the case. It is unclear to us how this sensitivity might be interpreted, although it may be relevant that the other indicators in those plots are quantities integrated over regions defined by composition criteria, whilst $\xi_{2.5}$ is evaluated at a fixed mass coordinate.

The binding energy of the outer core also occasionally suggests that the likelihood of a successful SN explosion could be 

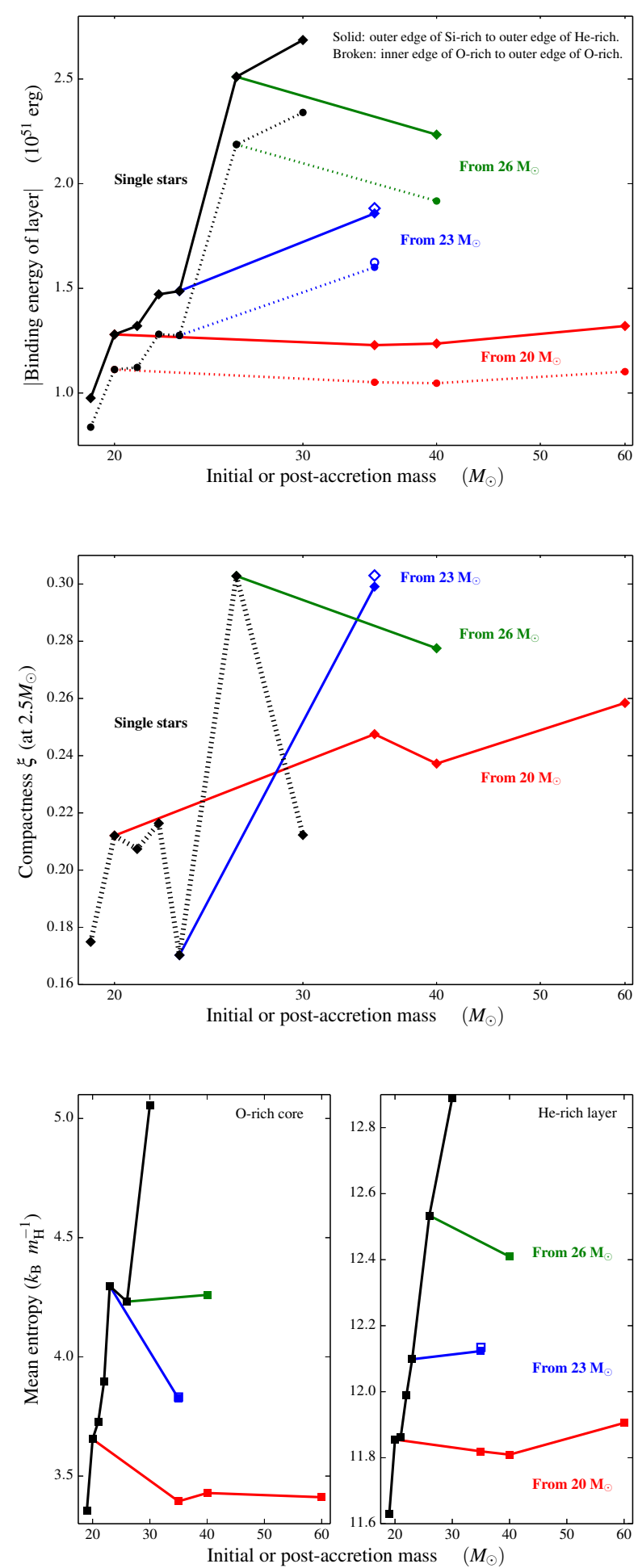

Figure 16. As Fig. 15 but for quantities at the end of core Si burning, where we do not show the oxygen core masses again. For almost all of these models, accretion began when the primary radius was $30 R_{\odot}$; the exception is plotted using hollow blue symbols, which represent a model for which accretion onto the primary began at $100 R \odot$. decreased by early Case B accretion, though less frequently than would be concluded from $\xi_{2.5}$. For the calculations with overshooting, accretion onto the $23 M_{\odot}$ primary increases the final binding energy (but not for post-accretion stars created from other primary masses). For the calculations without overshooting, the $50 M_{\odot}$ star formed from a $23 M_{\odot}$ primary shows an increase in final core binding energy compared to the $23 M_{\odot}$ single star (this is the same model which is an outlier to the trend for $\xi_{2.5}$ in that set of models).

\section{CHANNELS FOR EARLY CASE B ACCRETION: EVENT RATE CALCULATION AND COMPARISON}

In this section, we attempt to estimate the rates at which some relevant early Case B merger or accretion events are likely to occur. This section does not consider all possible formation channels, e.g., we make no attempt to account for dynamical mergers in dense young stellar clusters. However, in $\$ 5.3$, we discuss the potential importance of systems in which the early Case B merger was of the inner binary in a triple. In that case, the tertiary companion may be able to transfer even more mass onto the merger product before the SN explosion.

\subsection{Rates for the early Case B merger channel}

We now estimate the rate of luminous SNe that can be accounted for by our binary-merger model. When trying to explain CCSNe with immediate LBV progenitors using this scenario, two of the main uncertainties are the minimum postmerger mass required to produce the LBV phenomenology (since this governs the post-merger luminosity; see Figs. 2 \& 3) and the maximum pre-merger primary mass which can lead to a CCSN with canonical explosion energy. Assuming insignificant mass loss during the merger, then the maximum post-merger mass would be $\approx 1.8$ times the primary-star mass (due to the stability criteria for this early Case B merger process). So a $20 M_{\odot}$ primary may be able to attain a mass of $\approx 36 M_{\odot}$ after the merger, which is only just consistent with common expectations for the lower end of the range of initial LBV masses (although it has been suggested that even stars with initial masses as low as $25 M_{\odot}$ might display LBV-like phenomena, for which see Smith et al. 2004, Vink 2012). This estimate ignores any mass ejected during the merger, although post-merger rapid rotation may well increase the likelihood of LBV-type outbursts (Langer 1997, 1998). Given the uncertainties, we present estimates for a broad range of possible upper- and lower- limits on the primary-star mass.

We assume initial population properties guided by Kobulnicky \& Fryer (2007) and Sana et al. (2012). Table 1 presents those choices for the binary fraction $\left(f_{\text {binary }}\right)$ and initial period and mass-ratio distributions, along with the mass range of stars which is assumed to produce standard CCSNe (for normalising the LBV SN rate to the CCSN rate). We assume that all orbits are circular, and mostly use a massive-star IMF with a slope of -2.5 . This IMF slope is deliberately conservative. Whilst a flatter mass function has been inferred for the observed population of massive stars (a slope of -2.35 is typically adopted), that mass function may itself be caused by mergers of massive stars (i.e., mergers will tend to make the observed mass function flatter than the true initial mass function), as discussed by, e.g., Schneider et al. (2014) and references therein. Table 1 also contains some estimates for an IMF slope of -2.35 .

We feel that our adopted normalisation to the CCSN rate seems likely to be conservative. However, if the apparent upper mass limit for the progenitors of type IIP SNe is set by 

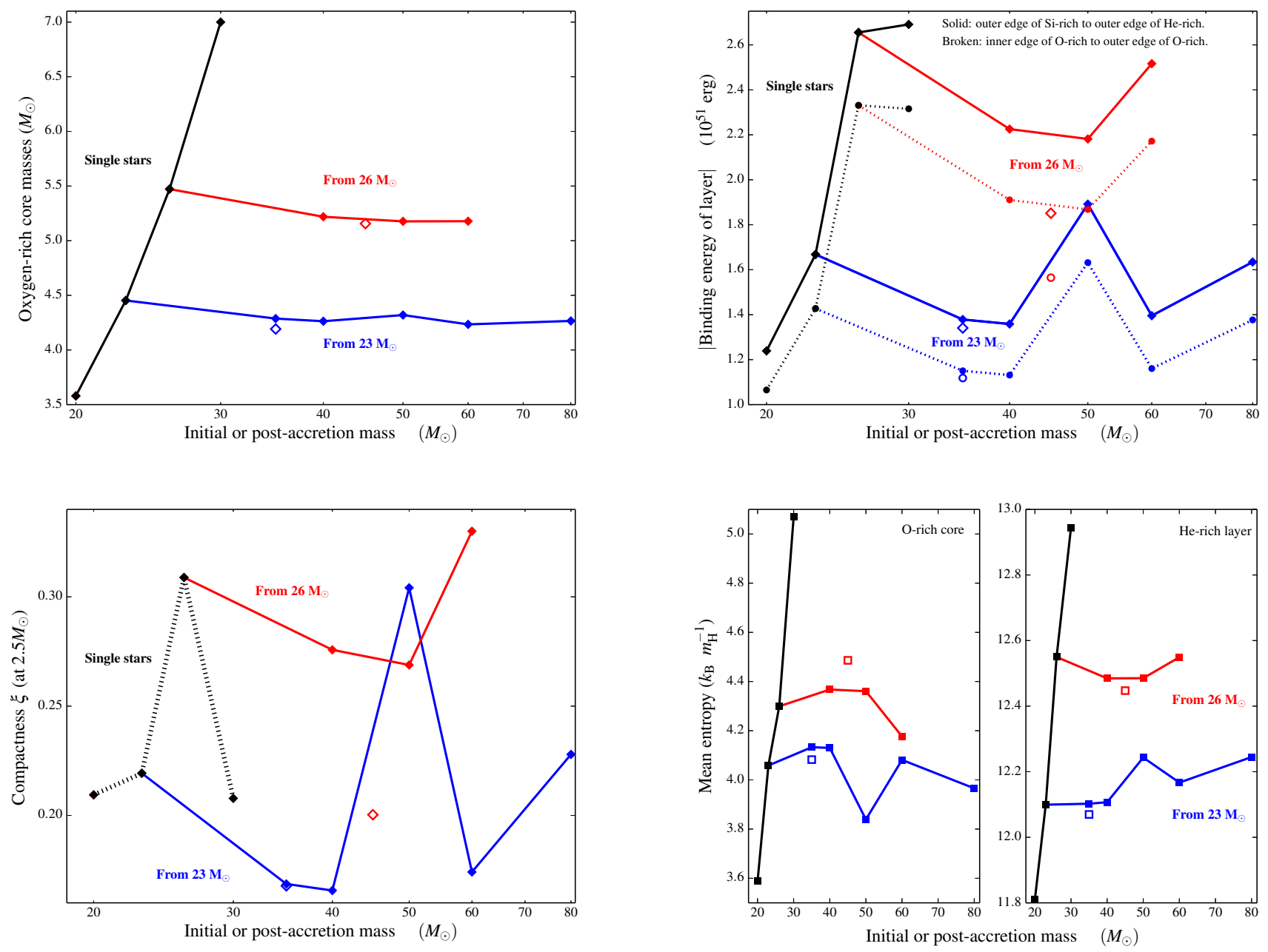

Figure 17. As Fig. 15 but for quantities at the end of central Si burning, and for calculations which assume the Schwarzschild criterion for convection without any overshooting. Here the solid symbols represent models for which accretion began at primary radii of $100 R_{\odot}$; the hollow symbols represent models for which accretion started when the primary had a radius of $30 R_{\odot}$.

the point at which stars form BHs (see, e.g., Kochanek 2014), then for any of our population models in which the upper limit for the CCSN normalisation is at a lower mass than the upper limit on the LBV SN primary mass, this requires the binary interactions to be able to cause the LBV progenitors to avoid $\mathrm{BH}$ formation at core collapse, or to somehow produce a luminous SN whilst forming a $\mathrm{BH}$. We have suggested that the former is plausible, but not proven it.

For most of our rate estimates we have assumed that the distribution of binary separations $a$ is flat in $\log (a)$, as is conventional. For these systems we also adopt a standard normalisation for the separation distribution, chosen under the general assumption that the range of $a$ runs from $3 R_{\odot}$ to $10^{4} R_{\odot}$ (for which see, e.g., Hurley et al. 2002). This is somewhat conservative, since such massive stars will not populate the region with $a$ as small as $3 R_{\odot}$; however this particular choice leads to only a relatively small underestimate. Changing the normalisation to one appropriate for $10<\left(a / R_{\odot}\right)<10^{4}$ would increase our predicted rates by only $\approx 15 \%$, whilst pessimistically taking $10<\left(a / R_{\odot}\right)<10^{5}$ would reduce the predicted rates by a similar amount.

Recent work using data from the VLT-FLAMES survey (Sana et al. 2012, 2013) has confirmed the expected high frac- tion of interacting binary stars within the massive-star population, but found binary properties for massive stars somewhat different to those assumed above. As is conventional, they used single power-law distribution functions to fit the population parameters (i.e., $f(x) \propto x^{a}$, where $x$ is the quantity of interest and $a$ is the exponent to be fitted). For their Galactic sample they found that the exponent of the $\log (P)$ distribution function is $0.55 \pm 0.22$ and that of the $q$ distribution function is $0.10 \pm 0.58$ (Sana et al. 2012), i.e., shorter orbital periods and lower-mass companions were both found to be more common than we adopted. We note that the constraint on the mass-ratio distribution in particular is fairly weak; the $f(q) \propto q$ distribution motivated by Kobulnicky \& Fryer (2007) is within $2 \sigma$ of these newer results. We also provide comparison rates using these parameters, assuming circular orbits.

The main source of uncertainty may be our lack of knowledge about exactly which binaries will merge after reaching contact inside the HG. It is qualitatively expected that donors with a steep density gradient in their envelopes (those with radiative envelopes) are more likely to lead to a merger than those with relatively shallow density profiles (those with deep convective envelopes). The phase during which we assume that the binary will merge is defined by the post-main- 
sequence expansion by a factor of 10 in radius (i.e., from the early- to mid-HG); this might easily be too conservative. Based on our earlier evolution calculations, we are confident that - if the systems merge during that phase - they would produce BSG SN progenitors.

In estimating these rates, we have assumed that no significant amount of mass is ejected during the merger. However, loss of material during the merger can be included in the rate estimates by appropriately increasing the minimum massratio limit for suitable mergers. For example, for a primary of $20 M_{\odot}$ at the time of the merger then, if $1 M_{\odot}$ is assumed to be ejected during the merger, then this corresponds to increasing the effective minimum mass ratio which can produce a suitable - i.e., sufficiently luminous - post-merger star from 0.6 to 0.65 .

Given all the above, we overall consider the rates presented in Fig. 18 and Table 11 likely to be conservative, although we admit that there are large uncertainties. Those estimated rates are typically in excess of one CCSN with an LBV progenitors per thousand CCSNe, in some cases approaching one per hundred CCSNe.

The observationally-derived rates are not precise for this class of SNe, but they can only be a subset of the SNe which display type IIn phenomenology (as discussed in \$1). Unfortunately, even the absolute rates of SN IIn are not certain. Based on our estimates, the rates for LBV SNe following mergers could easily be large enough to make a substantial contribution to the type IIn SN class. The rate of type IIn SNe is a small fraction of the overall CCSN rate (see, e.g., Kiewe et al. 2012). Pessimistically, we might only explain $1 \%$ of SN IIn (e.g., if SN IIn constitute $10 \%$ of CCSNe and our mergers produce only one $\mathrm{SN}$ in $1000 \mathrm{CCSNe}$ ). Conversely, if SN IIn produce only $2 \%$ of the volumetric CCSN rate, and only roughly half of those are from true CCSNe, then our more optimistic estimates for this formation channel could explain all of the genuinely core-collapse type IIn SNe.

The range of predicted rates for LBV SNe considerably exceeds the empirical rate for the superluminous $\mathrm{SNe}$ (which is estimated to be between $10^{-3}$ and $10^{-4}$ times the CCSN rate, see Tanaka et al. 2012). This is as qualitatively expected if special circumstances are necessary to lead to a radiativelyefficient $\mathrm{SNe}$, such as the ejection of a particularly massive shell just a few years before the explosion (Smith \& McCray 2007). However, we cannot be sure whether appropriate LBVtype mass-ejections would occur sufficiently often to account for the SLSNe in this way. Clearly it would help to support this model if such outbursts become more likely as these stars approach core collapse, perhaps by combining standard LBVlike instabilities with a driving mechanism similar to that proposed by Quataert \& Shiode (2012).

\subsubsection{What fraction of LBVS are this type of SN progenitor?}

It would be extremely difficult to give anything like a precise estimate for the present-day fraction of LBVs which were formed in this way. This is partly because LBVs may be formed through multiple binary channels in addition to the portion of the single-star IMF which produces LBVs. In addition, the duration of the LBV phase may well be different for LBVs which were formed through different routes. However, we can make a very rough estimate by comparing our predicted rates to the formation rate of single massive stars which are suitable massive to produce LBVs. To do this, we can compare a notional single-star LBV birthrate to a singlestar CCSN rate (similar to the normalisation used in Table 1 and Fig. 18 but here we simply integrate over different ranges of masses from a single-star IMF for the LBVs and CCSNe). Thereby we estimate that such single-star LBVs would form at roughly $10 \%$ of the rate at which a notional population of purely single-star CCSN would occur; this is accurate to within a factor of $\approx 2$ (in either direction) for a range of assumptions ${ }^{6}$ This suggests that LBVs formed from this particular merger channel - at $\lesssim 1 \%$ of the $\mathrm{CCSN}$ rate - constitute $\lesssim 10 \%$ of LBVs. We stress that this estimate neglects several potentially large factors. Nonetheless, we would be surprised if many more than $\sim 10 \%$ of present-day LBVs were to reach core collapse whilst still in the LBV phase.

\subsection{The stable mass transfer channel}

The early Case B merger channel is only one of the ways in which a star might potentially gain mass at the correct point in its evolution. That merger channel can naturally be triggered by the expansion of the primary at the appropriate point in its evolution (i.e., effectively the star gains mass upon expansion, which is the reverse of the normal expectation), which helps with fine-tuning the timing of the mass accretion. An alternative way in which a star could gain mass at a suitable time to become one of the BSG SN progenitors we model is if the star is the accreting secondary in a binary system in which the primary star happens to fill its Roche lobe when the secondary is in an appropriate phase of evolution. We will call this the stable mass-transfer formation channel.

If we require that the secondary has already left the main sequence when the primary transfers mass, then the qualitative conditions for this formation channel to operate are roughly similar to the conditions for "double-core evolution" to occur (see, e.g., Bethe \& Brown 1998; Dewi et al. 2006), but with a more restrictive limit on the evolutionary phase of the accretor. For double-core evolution, this mass transfer leads to an unstable contact phase, and thence to a special case of common-envelope evolution in which two cores spiral-in inside the shared envelope. The Galactic birthrate of binaries produced from double-core evolution has been variously estimated to be between $\sim 10^{-6} \mathrm{yr}^{-1}$ and $\sim 10^{-4} \mathrm{yr}^{-1}$ (see, e.g., Bethe \& Brown 1998; Dewi et al. 2006, and references therein). Only the upper end of those double-core birthrates are comparable to our estimates for the early Case B merger channel. Since the constraints on timing for this stable masstransfer channel are tighter than for double-core evolution, we conclude that production of these LBV SN progenitors will probably occur less frequently via stable mass transfer than through the early Case B merger channel.

\subsection{Post-merger accretion from the tertiary star in a triple}

Some of the post-accretion models shown earlier in this paper have been for post-accretion masses which could not be produced if the early Case B merger mechanism on which was have concentrated (and for which the rates in \$5.1 were derived) was the only way for the primary star to gain mass. One potential route through which unusually massive postmerger stars might be formed involves triple stars (see, e.g., Fig.4).

As an observational example, the massive binary system R4 in the Small Magellanic Cloud is presently best explained by

\footnotetext{
${ }^{6}$ For this we applied IMF exponents of both -2.5 and -2.35 . For CC$\mathrm{SNe}$, we tried combinations of minimum ZAMS masses between $8-10 M_{\odot}$ and maximum ZAMS masses between 20-30 $M_{\odot}$. For LBVs, we adopted minimum ZAMS masses between $35-45 M_{\odot}$ and maximum ZAMS masses of $100 M_{\odot}$ (the integral is not sensitive to the upper bound).
} 
Table 1

Population assumptions and associated rate estimates for the primordial binary early Case B merger channel alone.

\begin{tabular}{|c|c|c|c|c|c|c|c|c|c|c|}
\hline \multirow{2}{*}{$\begin{array}{l}\text { Name for } \\
\text { Fig. } 18\end{array}$} & \multicolumn{5}{|c|}{ Assumptions about initial population \& normalisation to CCSN } & \multicolumn{4}{|c|}{ Ranges assumed for scenario } & \multirow{2}{*}{$\begin{array}{l}\text { Rate estimate } \\
\log \left(\frac{\text { rate }}{\text { CCSNrate }}\right)\end{array}$} \\
\hline & $f_{\text {binary }}$ & $M_{1}$ dist. & $q$ dist. & $\log P$ dist. & $\mathrm{CCSN}_{\text {range }} \mathrm{a}^{\mathrm{a}}$ & $M_{1, \min }$ & $M_{1, \max }$ & $q_{\min } \mathrm{b}$ & Radius expansion $^{\mathrm{c}}$ & \\
\hline \multirow[t]{8}{*}{ KF1 } & $50 \%{ }^{d}$ & $\propto M_{1}^{-2.5}$ & $\propto q$ & flat & $8-40 M_{\odot}$ & 15 & 35 & 0.6 & 10 & -2.02 \\
\hline & & & & & & 20 & 35 & 0.6 & 10 & -2.31 \\
\hline & & & & & & 20 & 30 & 0.6 & 10 & -2.41 \\
\hline & & & & & & 20 & 25 & 0.6 & 10 & -2.61 \\
\hline & & & & & & 15 & 35 & 0.7 & 10 & -2.30 \\
\hline & & & & & & 20 & 35 & 0.7 & 10 & -2.59 \\
\hline & & & & & & 20 & 30 & 0.7 & 10 & -2.68 \\
\hline & & & & & & 20 & 25 & 0.7 & 10 & -2.89 \\
\hline \multirow[t]{32}{*}{ KF2 } & $70 \%{ }^{d}$ & $\propto M_{1}^{-2.5}$ & $\propto q$ & flat & $8-30 M_{\odot}$ & 15 & 35 & 0.6 & 10 & -1.88 \\
\hline & & & & & & 20 & 35 & 0.6 & 10 & -2.17 \\
\hline & & & & & & 20 & 30 & 0.6 & 10 & -2.30 \\
\hline & & & & & & 20 & 25 & 0.6 & 10 & -2.50 \\
\hline & & & & & & 15 & 35 & 0.7 & 10 & -2.15 \\
\hline & & & & & & 20 & 35 & 0.7 & 10 & -2.44 \\
\hline & & & & & & 20 & 30 & 0.7 & 10 & -2.57 \\
\hline & & & & & & 20 & 25 & 0.7 & 10 & -2.77 \\
\hline & & & & & & 15 & 35 & 0.6 & 30 & -1.71 \\
\hline & & & & & & 20 & 35 & 0.6 & 30 & -2.00 \\
\hline & & & & & & 20 & 30 & 0.6 & 30 & -2.13 \\
\hline & & & & & & 20 & 25 & 0.6 & 30 & -2.33 \\
\hline & $70 \%{ }^{d}$ & $\propto M_{1}^{-2.5}$ & $\propto q$ & flat & $8-40 M_{\odot}$ & 15 & 35 & 0.6 & 10 & -1.94 \\
\hline & & & & & & 20 & 35 & 0.6 & 10 & -2.23 \\
\hline & & & & & & 20 & 30 & 0.6 & 10 & -2.32 \\
\hline & & & & & & 20 & 25 & 0.6 & 10 & -2.52 \\
\hline & & & & & & 15 & 35 & 0.7 & 10 & -2.21 \\
\hline & & & & & & 20 & 35 & 0.7 & 10 & -2.50 \\
\hline & & & & & & 20 & 30 & 0.7 & 10 & -2.60 \\
\hline & & & & & & 20 & 25 & 0.7 & 10 & -2.80 \\
\hline & $70 \%{ }^{d}$ & $\propto M_{1}^{-2.35}$ & $\propto q$ & flat & $8-30 M_{\odot}$ & 15 & 35 & 0.6 & 10 & -1.84 \\
\hline & & & & & & 20 & 35 & 0.6 & 10 & -2.12 \\
\hline & & & & & & 20 & 30 & 0.6 & 10 & -2.25 \\
\hline & & & & & & 20 & 25 & 0.6 & 10 & -2.46 \\
\hline & & & & & & 15 & 35 & 0.7 & 10 & -2.12 \\
\hline & & & & & & 20 & 35 & 0.7 & 10 & -2.40 \\
\hline & & & & & & 20 & 30 & 0.7 & 10 & -2.53 \\
\hline & & & & & & 20 & 25 & 0.7 & 10 & -2.74 \\
\hline & & & & & & 15 & 35 & 0.6 & 30 & -1.68 \\
\hline & & & & & & 20 & 35 & 0.6 & 30 & -1.96 \\
\hline & & & & & & 20 & 30 & 0.6 & 30 & -2.09 \\
\hline & & & & & & 20 & 25 & 0.6 & 30 & -2.29 \\
\hline \multirow[t]{20}{*}{ S1 } & $70 \% \mathrm{e}^{\mathrm{e}}$ & $\propto M_{1}^{-2.5}$ & $\propto q^{-0.1}$ & $\propto(\log P)^{-0.55}$ & $8-40 M_{\odot}$ & 15 & 35 & 0.6 & 10 & -1.92 \\
\hline & & & & & & 20 & 35 & 0.6 & 10 & -2.21 \\
\hline & & & & & & 20 & 30 & 0.6 & 10 & -2.30 \\
\hline & & & & & & 20 & 25 & 0.6 & 10 & -2.50 \\
\hline & & & & & & 15 & 35 & 0.7 & 10 & -2.23 \\
\hline & & & & & & 20 & 35 & 0.7 & 10 & -2.52 \\
\hline & & & & & & 20 & 30 & 0.7 & 10 & -2.61 \\
\hline & & & & & & 20 & 25 & 0.7 & 10 & -2.81 \\
\hline & $70 \% \mathrm{e}^{\mathrm{e}}$ & $\propto M_{1}^{-2.5}$ & $\propto q^{-0.1}$ & $\propto(\log P)^{-0.55}$ & $8-25 M_{\odot}$ & 15 & 35 & 0.6 & 10 & -1.83 \\
\hline & & & & & & 20 & 35 & 0.6 & 10 & -2.12 \\
\hline & & & & & & 20 & 30 & 0.6 & 10 & -2.23 \\
\hline & & & & & & 20 & 25 & 0.6 & 10 & -2.45 \\
\hline & & & & & & 15 & 35 & 0.7 & 10 & -2.14 \\
\hline & & & & & & 20 & 35 & 0.7 & 10 & -2.43 \\
\hline & & & & & & 20 & 30 & 0.7 & 10 & -2.54 \\
\hline & & & & & & 20 & 25 & 0.7 & 10 & -2.76 \\
\hline & & & & & & 15 & 35 & 0.6 & 30 & -1.67 \\
\hline & & & & & & 20 & 35 & 0.6 & 30 & -1.96 \\
\hline & & & & & & 20 & 30 & 0.6 & 30 & -2.06 \\
\hline & & & & & & 20 & 25 & 0.6 & 30 & -2.28 \\
\hline
\end{tabular}

${ }^{\text {a }}$ For normalisation to the CCSN rate. Secondary stars are included in the normalisation using the same mass-ratio distribution and $f_{\text {binary }}$.

b In all cases, $q_{\max }=0.8$.

c i.e. the range of radius expansion of the primary star after the end of the main sequence over which the outcome may be a merger.

d With separations $a$ such that $3 \leq\left(a / R_{\odot}\right) \leq 10^{4}$.

e With separations $a$ such that $3 \leq\left(a / R_{\odot}\right) \leq 5 \times 10^{3}$. 

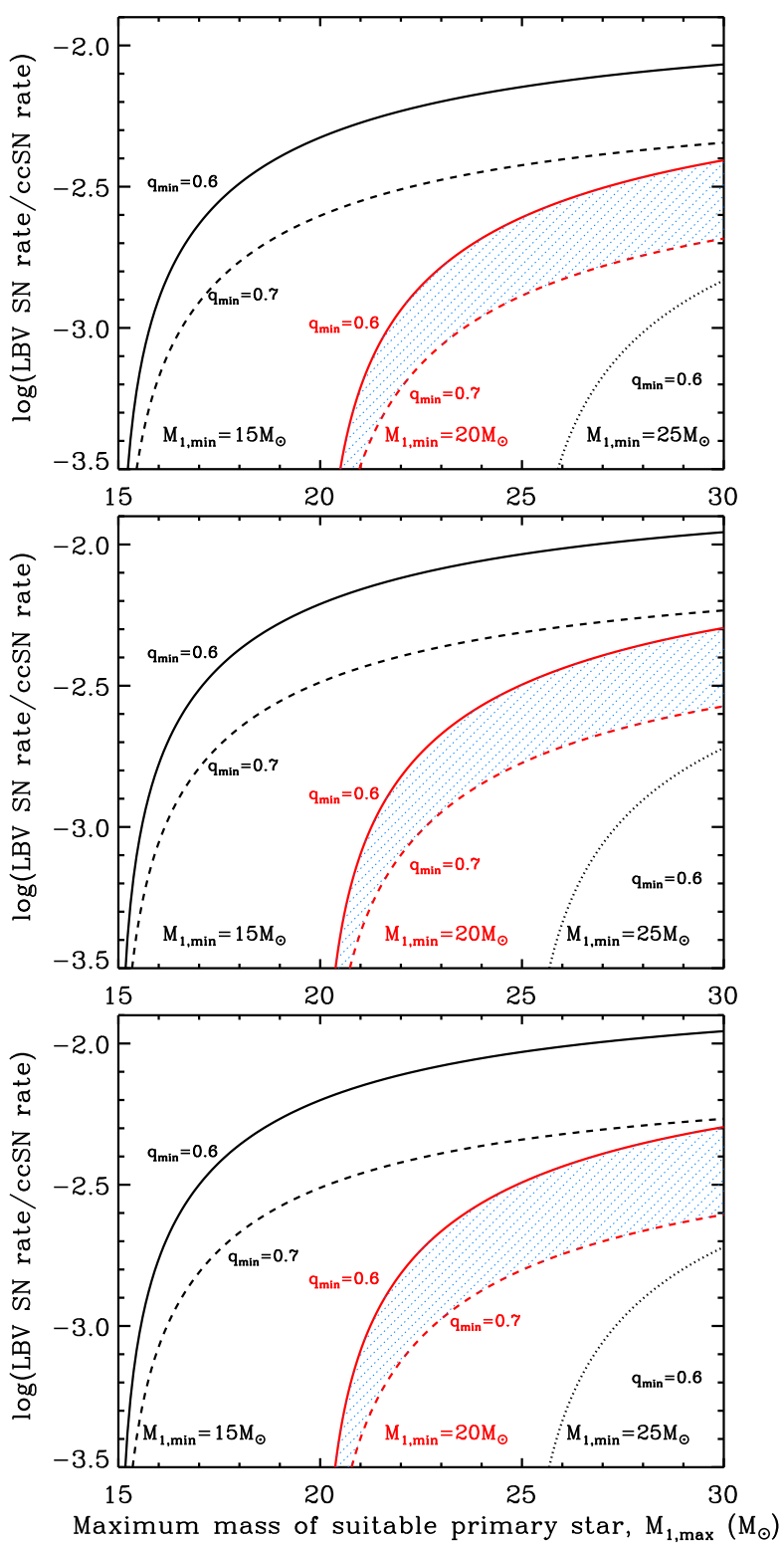

Figure 18. Estimated rates for $\mathrm{SNe}$ from our merger scenario, given as a fraction of the CCSN rate. Initial population assumptions are given in Table 1 (models KF1, KF2 and S1 are in the top, middle and bottom panels, respectively). All panels assume that a merger does not occur if the binary mass ratio ( $q$; accretor mass over donor mass) is higher than 0.8 . The minimum mass ratio for merger, $q_{\min }$, is 0.6 or 0.7 , as marked. The curves which bound the shaded regions assume that the primary needs to be more massive than $20 M_{\odot}$; those shaded regions therefore indicate a range of rate estimates for that assumption. More extreme cases are provided by the black curves, which assume that the primary needs to be more massive than only $15 M_{\odot}$ (solid and dashed curves) or at least $25 M_{\odot}$ (dotted curves).

a merger in a primordial triple (Pasquali et al. 2000). The current $\mathrm{B}[\mathrm{e}]$ star in $\mathrm{R} 4$ appears younger than the A star companion, suggesting that it was rejuvenated by a merger; that event could also lead to the nebula around the system (Pasquali et al. 2000). Some triples - similar to R4, though with a smaller separation of the remaining post-merger binary - might subsequently experience either a second merger, or mass transfer from the triple onto the merger product. In either case, such systems could produce a qualitatively different population of $\mathrm{SN}$ progenitors than those which involved only one merger

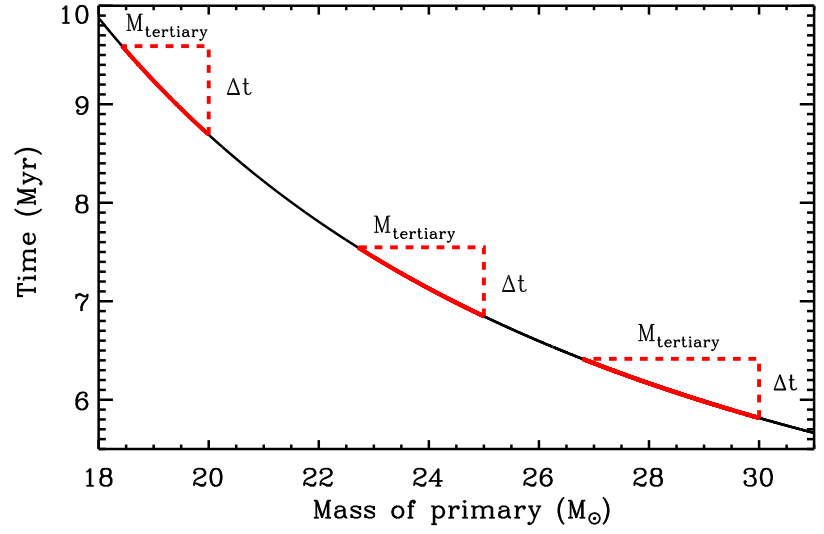

Figure 19. We estimate the range of tertiary masses for which the outer star in a "potentially-interacting" triple would evolve to fill its Roche lobe during the lifetime of the merger product. The black curve shows an approximate measure of the stellar lifetime before expanding to become a RSG $\left(t_{\mathrm{BGB}}\right.$ from the fits of Hurley et al. 2000 for a metallicity of 0.02 ; note that for our estimates here the absolute value is irrelevant, only the slope). We compare this to the rough lifetime of the merger product (denoted $\Delta t$ ) for three primary masses $\left(20,25\right.$ and $30 M_{\odot}$, for which we take post-merger lifetimes of 0.9, 0.7 and 0.6 Myr respectively, based on Fig. 5. Even though the post-merger lifetime decreases for increasing primary mass, the range of potentially-suitable tertiary masses $\left(M_{\text {tertiary }}\right)$ increases at higher primary masses (because the gradient of the curve decreases).

Table 2

The estimated fraction of potentially-interacting triples which, if the inner binary produces a merger, possess a mass ratio which leads to mass transfer onto the merger product.

\begin{tabular}{|c|c|c|c|c|c|}
\hline \multirow[b]{2}{*}{$M_{1}$} & \multirow[b]{2}{*}{$\min \left(M_{3}\right)$} & \multirow[b]{2}{*}{$\max (q)$} & \multicolumn{3}{|c|}{$\begin{array}{l}\text { Fraction of interacting triples suitable, } \\
\text { for a } q \text {-distribution with exponent: }\end{array}$} \\
\hline & & & 0 (i.e., flat) & 1 (i.e., correlated) & -0.1 \\
\hline \multirow[t]{3}{*}{20} & 18.43 & 1.0 & 0.079 & 0.151 & 0.071 \\
\hline & & 0.99 & 0.069 & 0.131 & 0.062 \\
\hline & & 0.95 & 0.029 & 0.054 & 0.026 \\
\hline \multirow[t]{3}{*}{25} & 22.71 & 1.0 & 0.092 & 0.175 & 0.083 \\
\hline & & 0.99 & 0.082 & 0.155 & 0.074 \\
\hline & & 0.95 & 0.042 & 0.077 & 0.038 \\
\hline \multirow[t]{3}{*}{30} & 26.79 & 1.0 & 0.107 & 0.202 & 0.097 \\
\hline & & 0.99 & 0.097 & 0.183 & 0.088 \\
\hline & & 0.95 & 0.057 & 0.105 & 0.052 \\
\hline
\end{tabular}

(or accretion phase). These stars would also have little time to lose the angular momentum gained during their second accretion phase.

Overall rate estimates for triple stars are even more uncertain than for binary stars. However, we can estimate the fraction of triples in which the tertiary star would expand away from the main sequence after the inner binary has merged but before the merger product explodes as a SN by using the post-merger lifetimes shown in Fig. 5. We will call triples in which the tertiary orbit is small enough for the tertiary to fill its Roche lobe at some point in its evolution "potentially interacting" (since the triple would not have the chance to interact if the system has been disrupted by a SN before the tertiary expands to fill its Roche lobe). We note that mass transfer from the outer star in a triple onto the inner binary has been discussed by Tauris \& van den Heuvel (2014) and de Vries et al. (2014), although in very different contexts. Perets \& Fabrycky (2009) have also considered how triple stars may be 
important in promoting blue-straggler formation.

Fig. 19 demonstrates how we estimate the potentiallysuitable range of tertiary masses. Given those mass ranges, we can then estimate the fraction of "potentially interacting" triples which meet the necessary criteria. These estimates are shown in Table 2 for different assumptions about the distribution of the mass of the tertiary star relative to the primary star. Table 2 also shows how those estimates change if we limit the mass of the outer triple to be less than $99 \%$ or $95 \%$ of the mass of the primary. For all but the more conservative sets of assumptions, these estimates indicate that $\approx 10$ percent of the "potentially interacting" triples in which the inner binary is subject to an early Case B merger would produce accretion onto the merger product.

Whilst we do not claim to properly estimate the fraction of O-stars which are in such "potentially interacting" triples, we stress that O-stars are very commonly found in triple systems. Eggleton \& Tokovinin (2008) found that the combined fraction of O-star systems with triple or higher multiplicity was higher than the binary fraction of O-star systems (the fraction of O-star systems which they detected to have triple multiplicity was $\approx 2 / 3$ of the binary fraction; note that this does not include the systems with higher multiplicity which could contain a suitable triple-star sub-system) ${ }^{7}$ We therefore consider it realistic that as many as $\sim 10 \%$ of the early Case-B mergers might potentially gain further mass from a tertiary star. Somewhat less than $10 \%$ of our binary merger rate (from $\$ 5.1$ ) would produce a $\mathrm{SN}$ rate that matches the observationallyinferred rate of SLSN-II. This might well be coincidence, but nonetheless suggests that further study of this evolutionary channel is deserved.

\section{DISCUSSION}

\subsection{The relationship between "normal" SNe with $L B V$ progenitors and "superluminous" SNe.}

The calculations presented in this paper have shown that early Case B accretion is able to produce BSGs which are plausibly sufficiently luminous to have been LBVs immediately before core collapse. Our rate estimates further indicate that the birthrate from the early Case B binary-merger scenario may well be high enough to explain the CCSNe of normal luminosity which have been inferred to have immediate LBV progenitors. Some particularly luminous LBV progenitors of CCSNe might be explained by a second phase of mass accretion from a tertiary companion, or potentially by multiple mergers in dense stellar systems.

However, this does not guarantee that this class of formation channels can explain any of the progenitors of SLSNe. If the hypothesis is correct that some SLSNe can be explained by greater radiative efficiency of a $\mathrm{SN}$ with otherwise normal energetics, then this might be explained by CSM produced by an appropriately-timed and appropriately-massive LBV outburst. This still appears to be a reasonable model for at least a subset of the SLSNe, given that the amount of CSM required to lead to radiatively-efficient events is disputed (see, e.g., Dwarkadas 2011). However, it might be that normal LBV outbursts are incapable of producing the CSM properties which are necessary to explain SLSNe.

\subsection{Metallicity effects}

\footnotetext{
${ }^{7}$ It also seems plausible that the fraction of the "potentially interacting" triples in which the inner binary merges is higher than for standard binaries.
}

Our models have all assumed a metallicity of $\mathrm{Z}=0.02$. A priori, it is unclear what effect changing this assumption would have on our results. If the initial binary-star population does not vary as a function of metallicity, then we consider that moderate changes in metallicity should not lead to a significant change in the rate at which suitable mergers occur. However, for metallicities which are low enough that the primary star burns He as a BSG, then the parameter space for early Case B mergers would be reduced.

Since it is broadly expected that LBV formation requires higher masses at lower metallicities (since the HumphreysDavidson limit moves to higher luminosities), then the parameter space for our merger model which is capable of forming LBV progenitors of CCSNe may be reduced.

Those potential effects on the rates are independent from any effects on the post-accretion structures. We hope to extend this study of stellar structures and evolution following early Case B mergers with a systematic future exploration of potential metallicity effects on the superficial appearance and the fate of the core, alongside an investigation of the potential effect of He-enrichment during the merger.

\subsection{Mergers of $q \approx 1$ massive binaries and potential pair-instability $\mathrm{SNe}$}

This paper has mainly concentrated on a scenario which involves a particular early Case B merger process in which the merger instability follows a brief contact phase. Those mergers are only expected to happen for a limited range of mass ratios, in particular with an upper limit on the mass ratio $(q<0.8)$. We repeat that these mergers are not a result of the canonical high-mass-ratio dynamical instabilities. There is an additional part of parameter space which is expected to lead to an unstable contact phase and binary mergers: the postmain-sequence merger of two stars of almost equal mass (i.e., $q \approx 1$ ).

Early Case B mergers from this channel would require finetuning, in that the masses would have to be so similar that both stars are expanding across the Hertzsprung gap at the same time (although massive stars may prefer similar-mass companions; see, e.g., Kobulnicky \& Fryer 2007). In most cases, this 'double-core' merger channel seems likely to lead to merged $\mathrm{He}$ cores and thence the formation of a $\mathrm{BH}$ at core collapse, even if the star is then an LBV.

However, mergers of sufficiently massive post-mainsequence cores should form massive enough oxygen cores to produce a pair-instability explosion (Barkat et al. 1967; Rakavy \& Shaviv 1967; Heger et al. 2003). This would not necessarily require an early Case-B merger, as long as both cores were adequately evolved. Predicted minimum $\mathrm{He}$ core masses for producing a pair-instability $\mathrm{SN}$ are $\approx 64 M_{\odot}$ (Heger et al.2003), which suggests that this scenario requires a merger of two stars each of initial mass in excess of $\approx$ $50 M_{\odot}$ (with some uncertainty coming from the treatment of convection in the cores of such stars; note that the fractional core mass increases with stellar mass). Unlike standard pair-instability events, this may well occur even at solar metallicity. (See also Pan et al.2012, who noted that runaway collisions in stellar clusters could also help to generate pairinstability $\mathrm{SNe}$ at solar metallicity.) Furthermore, in very finetuned cases these merger-produced pair-instability SNe might potentially take place inside a recently-ejected (or partiallyejected) envelope. If such fine-tuning is possible then this could lead, in principle, to an unusually energetic and unusually radiatively-efficient $\mathrm{SN}$. 


\subsection{Tertiary-star CE ejection}

Another route through which a SN might happen inside a recently-ejected stellar envelope is a variation of the triplestar scenario described in $\$ 5.3$. Since we find that the merger products expand very late in their nuclear evolution, that postHe-core-burning expansion might trigger the onset of a $\mathrm{CE}$ phase within $10 \mathrm{kyr}$ of core collapse. This may occur if the expanding merger product is already accreting from a tertiary star (leading to an unstable contact phase), or if the expansion leads to dynamically-unstable Roche-lobe overflow onto a tertiary companion (although this may well require fine-tuning of the separations in the initial triple system). In some cases the time to core collapse could be comparable to the potential combined duration of the onset and spiral-in of the $\mathrm{CE}$ phase, i.e., a CCSN might occur inside many solar masses of ejected material. The outcome would be similar to the model of Chevalier (2012), although this post-merger scenario may help to explain why the timing of the ejection was so close to core collapse.

\subsection{Observables and tests of the model}

Our post-merger BSG models have lower core mass fractions than canonical main-sequence BSGs. The difference is even larger when compared to LBV SN models which invoke rotation-induced mixing, which have cores which account for almost the entirety of the stellar mass (Groh et al. 2013). Unfortunately, it is not clear whether this difference can be practically tested. The core masses might perhaps be inferred by means of asteroseismology if oscillation modes of the stars are sufficiently excited (see, e.g., Saio et al. 2013). As we expect these BSGs to spend time as $\mathrm{B}[\mathrm{e}]$ supergiants (Podsiadlowski et al. 2006), we encourage attempts to determine the structures of $\mathrm{sgB}[\mathrm{e}]$ stars. Alternatively, perhaps reconstruction of the structure of the progenitor star from a suitable SN would enable discrimination between the possibilities, either by using time-resolved spectroscopy (e.g., Mazzali et al. 2008) or analysis of nebular spectra (e.g., Mazzali et al. 2010).

Other observables during the BSG/LBV phase might have multiple interpretations. For example, the bipolar shell surrounding the LBV candidate $\mathrm{G} 25.5+0.2$ might well have been produced by ejecta from a stellar merger, and the projected peak expansion velocities of the nebula $\left(\sim 180 \mathrm{~km} \mathrm{~s}^{-1}\right)$ are comparable to surface escape velocities from HG stars (Clark et al.2000). However, even if one could prove that the nebula was generated by a merger, it is unclear how one could unambigously determine whether the merger in that particular system was during Case B. Likewise, we would not be surprised to find surface abundance anomalies in the envelope of a post-merger BSG/LBV, such as enhancement in helium or nitrogen, but conclusions drawn from observations of such enrichment may not be definitive (for a discussion of how surface nitrogen abundances and rotational velocities might help to constrain BSG properties see, e.g, Vink et al. 2010).

Clearer surface observables might be provided during the post-accretion/post-merger contraction phase, but the duration of this stage of the evolution is relatively brief. This phase is also the one most likely to have its appearance affected by the details of the merger physics, so it is the one for which quantitative predictions are the most uncertain. Nonethless, the qualitative changes in our models during this phase have similarities to the rapid temperature increases seen in Eta Carinae since the Great Eruption (Rest et al. 2012; Mehner et al.2014). We intend to explore this similarity in the future.

\section{CONCLUSIONS}

We have explored the results of early Case B accretion, concentrating on primary-star masses which are at the upper end of the range which seem likely to produce NSs after core collapse. We find that, if massive stars are able to gain sufficient mass soon after they finish core $\mathrm{H}$ burning, they can reach core collapse with the properties expected of an LBV. We have demonstrated that our results are robust against some reasonable variations of the stellar physics employed.

The amount of accretion which is required to produce LBV SN progenitors from such primary stars might be supplied by early Case B mergers following an unstable contact phase. Our estimates for the birthrate from this merger channel are broadly consistent with the inferred rate of LBV SNe, at more than one per thousand CCSNe and approaching 1 per cent of the CCSN rate for moderately optimistic assumptions.

Additional contributions to the birthrate may be obtained through other formation channels, notably from very late Case A contact binaries which merge after the primary leaves the main sequence. Or in some rare cases, a RSG primary may fill its Roche-lobe and transfer its envelope to the secondary just as the secondary is leaving the MS (see $\$ 5.2$ ). In dense stellar environments, well-timed direct collisions might potentially account for some similar SN progenitors, including some examples in which even more mass could be accreted than is possible for co-eval field binary evolution. We have calculated evolutionary tracks to examine the evolution of stars which accrete more mass than simple binary evolution should allow, but we have not attempted to estimate the rate at which such merger products might be produced.

Surprisingly, stable mass transfer from a tertiary star onto the product of an early Case B merger may be an important channel for the formation of some extreme SN progenitors. Indeed, rough estimates indicate that this channel might be more common than early Case B accretion from stable masstransfer in a binary. This assumes that mass transfer onto the merger product can occur at any time during the lifetime of the merger product, which requires less fine-tuning than that which is required to achieve mass transfer onto a star in the Hertzsprung Gap.

We have also investigated whether the core collapse of these post-accretion stars is likely to lead to a successful SN explosion. To study this we have compared the core structures of the post-accretion stars to those of single stars using a range of potential indicators: the mass of the $\mathrm{CO}$ core, the binding energy of the outer core, the compactness parameter $\xi_{2.5}$ and the mean specific entropy of the core (for both the $\mathrm{CO}$ core and the He-rich layer outside the CO core). These generally suggest that early Case B accretion onto the envelope of a star does not significantly increase the likelihood of $\mathrm{BH}$ formation at core collapse. However, $\xi_{2.5}$ leads to ambiguous predictions: for $\xi_{2.5}$ the predicted effect of accretion differs between our set of models which adopt significant overshooting and the set which assumes the Schwarzschild criterion with no overshooting. Moreover, we suggest that when there are changes in the final core properties, these are more often in the direction of the core becoming more similar to the core of a lower-mass star, not a higher-mass one. Despite the uncertainties arising from the assumptions about the accretion phase (or merger process), the fact that accretion may increase the chance of a successful CCSN is striking. If this result is confirmed then this effect may be very significant in understanding the diversity of CCSNe from binary progeni- 
tors.

However, the merger products often display a combination of core properties which are not possessed by any of our single-star models. The fact that binary interactions can affect observed SN diversity by changing the distributions of final envelope masses and final core angular momenta has long been appreciated (see, e.g., Podsiadlowski et al. 1992). The effects of binary interactions on the structure of the final core have been less widely studied (but see, e.g., Podsiadlowski et al. 2004; Poelarends et al. 2008). Our results add to the evidence that the core collapse of non-rotating stars at fixed metallicity may be poorly described by a single-parameter family in He core mass. They also strengthen the idea that binary interactions are vital for understanding the diversity of CCSNe. In future we plan to improve the density of our model coverage within the binary parameter space. We also intend to study the physics of the merger process, and the potential effects of that merger physics on the post-merger evolution.

The recently-recognised explosions of LBV stars have sometimes been presented as a challenge to existing theories of stellar evolution. In contrast, a class of binary mergers is able to produce events which naturally match the inferred properties of the relevant SN progenitors. This one channel may be able to produce a diverse range of $\mathrm{SN}$ types, ranging from the explosions of yellow supergiants to superluminous $\mathrm{SNe}$. These SNe and the stellar mergers which preceded them are extraordinary in their physical and astrophysical interest and deserve greater theoretical attention.

\section{ACKNOWLEDGEMENTS}

The final form of this work has been significantly influenced by an anonymous referee whom we thank for a critical examination which prompted numerous improvements. SJ thanks the Chinese Academy of Sciences and National Science Foundation of China for support (grant numbers 11250110055 and 11350110324). SJ and PhP thank Zhanwen Han for generous hospitality, Ilka Petermann for useful discussions, and the attendees at the "Fireworks 2012" workshop for helpful comments. PhP and JSV thank the Aspen Center for Physics (and NSF Grant number 1066293) for hospitality during discussions of parts of this work. The authors are very grateful to Peter Eggleton, Bill Paxton, and their respective collaborators for their hard work and for making their stellar evolution packages available. This publication has made use of matplotlib (Hunter 2007).

\section{REFERENCES}

Abt, H. A., \& Levy, S. G. 1976, ApJS, 30, 273

-. 1978, ApJS, 36, 241

Aldering, G., et al. 2006, ApJ, 650, 510

Anderson, J. P., Habergham, S. M., James, P. A., \& Hamuy, M. 2012, MNRAS, 424, 1372

Barkat, Z., Rakavy, G., \& Sack, N. 1967, Physical Review Letters, 18, 379 Barkat, Z., \& Wheeler, J. C. 1988, ApJ, 332, 247

-. 1989, ApJ, 341, 925

Belczynski, K., \& Taam, R. E. 2008, ApJ, 685, 400

Berger, E., Foley, R., \& Ivans, I. 2009, The Astronomer's Telegram, 2184, 1

Bethe, H. A., \& Brown, G. E. 1998, ApJ, 506, 780

Braun, H., \& Langer, N. 1995, A\&A, 297, 483

Brown, G. E., \& Bethe, H. A. 1994, ApJ, 423, 659

Brown, G. E., Heger, A., Langer, N., Lee, C.-H., Wellstein, S., \& Bethe, H. A. 2001, New A, 6, 457

Brown, G. E., \& Lee, C.-H. 2004, New A, 9, 225

Brown, G. E., Lee, C.-H., Wijers, R. A. M. J., \& Bethe, H. A. 2000

Phys. Rep., 333, 471

Bruenn, S. W., et al. 2013, ApJ, 767, L6

Chatzopoulos, E., \& Wheeler, J. C. 2012a, ApJ, 748, 42

-. 2012b, ApJ, 760, 154
Chen, K.-J., Woosley, S., Heger, A., Almgren, A., \& Whalen, D. J. 2014, ApJ, 792, 28

Chevalier, R. A. 2012, ApJ, 752, L2

Chugai, N. N., \& Danziger, I. J. 1994, MNRAS, 268, 173

Chugai, N. N., et al. 2004, MNRAS, 352, 1213

Clark, J. S., Steele, I. A., \& Langer, N. 2000, ApJ, 541, L67

Colgate, S. A., \& White, R. H. 1966, ApJ, 143, 626

Couch, S. M., \& O'Connor, E. P. 2014, ApJ, 785, 123

de Mink, S. E., Langer, N., Izzard, R. G., Sana, H., \& de Koter, A. 2013, ApJ, 764, 166

de Mink, S. E., Sana, H., Langer, N., Izzard, R. G., \& Schneider, F. R. N. 2014, ApJ, 782, 7

de Vries, N., Portegies Zwart, S., \& Figueira, J. 2014, MNRAS, 438, 1909

Dewi, J. D. M., Podsiadlowski, P., \& Sena, A. 2006, MNRAS, 368, 1742

Dilday, B., et al. 2012, Science, 337, 942

Dopita, M. A., Cohen, M., Schwartz, R. D., \& Evans, R. 1984, ApJ, 287, L69

Drake, A. J., et al. 2010, ApJ, 718, L127

Dwarkadas, V. V. 2011, MNRAS, 412, 1639

Eggleton, P. P. 1971, MNRAS, 151, 351

- 1972, MNRAS, 156, 361

-. 1973, MNRAS, 163, 279

-. 2010, New A Rev., 54, 45

Eggleton, P. P., Faulkner, J., \& Flannery, B. P. 1973, A\&A, 23, 325

Eggleton, P. P., \& Tokovinin, A. A. 2008, MNRAS, 389, 869

Ekström, S., et al. 2012, A\&A, 537, A146

Eldridge, J. J., \& Tout, C. A. 2004, MNRAS, 353, 87

Eldridge, J. J., \& Vink, J. S. 2006, A\&A, 452, 295

Fernández, R., Müller, B., Foglizzo, T., \& Janka, H.-T. 2014, MNRAS, 440, 2763

Fraser, M., et al. 2010, ApJ, 714, L280

-. 2013, MNRAS, 433, 1312

Fryer, C. L. 1999, ApJ, 522, 413

_. 2013, Classical and Quantum Gravity, 30, 244002

Fryer, C. L., \& Heger, A. 2000, ApJ, 541, 1033

Fryer, C. L., Heger, A., Langer, N., \& Wellstein, S. 2002, ApJ, 578, 335

Gal-Yam, A. 2012, Science, 337, 927

Gal-Yam, A., \& Leonard, D. C. 2009, Nature, 458, 865

Gal-Yam, A., et al. 2007, ApJ, 656, 372

-. 2009, Nature, 462, 624

Gezari, S., et al. 2009, ApJ, 690, 1313

Glatzel, W., \& Kiriakidis, M. 1993, MNRAS, 263, 375

Glebbeek, E., Gaburov, E., de Mink, S. E., Pols, O. R., \& Portegies Zwart, S. F. 2009, A\&A, 497, 255

Glebbeek, E., Gaburov, E., Portegies Zwart, S., \& Pols, O. R. 2013,

MNRAS, 434, 3497

Gräfener, G., Owocki, S. P., \& Vink, J. S. 2012, A\&A, 538, A40

Groh, J. H., Meynet, G., \& Ekström, S. 2013, A\&A, 550, L7

Groh, J. H., et al. 2009, ApJ, 705, L25

Guzik, J. A., \& Lovekin, C. C. 2014, ArXiv e-prints: 1402.0257

Habergham, S. M., Anderson, J. P., James, P. A., \& Lyman, J. D. 2014 MNRAS, 441, 2230

Hamuy, M., et al. 2003, Nature, 424, 651

Hanke, F., Müller, B., Wongwathanarat, A., Marek, A., \& Janka, H.-T. 2013, ApJ, 770, 66

Heger, A., Fryer, C. L., Woosley, S. E., Langer, N., \& Hartmann, D. H. 2003, ApJ, 591, 288

Heger, A., Jeannin, L., Langer, N., \& Baraffe, I. 1997, A\&A, 327, 224

Heger, A., \& Langer, N. 1998, A\&A, 334, 210

Heger, A., Langer, N., \& Woosley, S. E. 2000, ApJ, 528, 368

Heger, A., Woosley, S. E., \& Spruit, H. C. 2005, ApJ, 626, 350

Herant, M., Benz, W., Hix, W. R., Fryer, C. L., \& Colgate, S. A. 1994, ApJ, 435,339

Herwig, F. 2000, A\&A, 360, 952

Hillebrandt, W., \& Meyer, F. 1989, A\&A, 219, L3

Hjellming, M. S., \& Webbink, R. F. 1987, ApJ, 318, 794

Howell, D. A., et al. 2013, ApJ, 779, 98

Humphreys, R. M., \& Davidson, K. 1994, PASP, 106, 1025

Hunter, J. D. 2007, Computing In Science \& Engineering, 9, 90

Hurley, J. R., Pols, O. R., \& Tout, C. A. 2000, MNRAS, 315, 543

Hurley, J. R., Tout, C. A., \& Pols, O. R. 2002, MNRAS, 329, 897

Inserra, C., et al. 2013, ApJ, 770, 128

Ivanova, N., Justham, S., Avendano Nandez, J. L., \& Lombardi, J. C. 2013, Science, 339, 433

Ivanova, N., \& Podsiadlowski, P. 2003, in From Twilight to Highlight: The Physics of Supernovae, ed. W. Hillebrandt \& B. Leibundgut, 19-+

Janka, H.-T., Langanke, K., Marek, A., Martínez-Pinedo, G., \& Müller, B. 2007, Phys. Rep., 442, 38

Joss, P. C., Salpeter, E. E., \& Ostriker, J. P. 1973, ApJ, 181, 429

Kasen, D., \& Bildsten, L. 2010, ApJ, 717, 245

Kasen, D., Woosley, S. E., \& Heger, A. 2011, ApJ, 734, 102

Kiewe, M., et al. 2012, ApJ, 744, 10

Kobulnicky, H. A., \& Fryer, C. L. 2007, ApJ, 670, 747

Kochanek, C. S. 2014, ApJ, 785, 28

Kochanek, C. S., Beacom, J. F., Kistler, M. D., Prieto, J. L., Stanek, K. Z.,

Thompson, T. A., \& Yüksel, H. 2008, ApJ, 684, 1336 
Kotak, R., Meikle, W. P. S., Adamson, A., \& Leggett, S. K. 2004, MNRAS, 354, L13

Kotak, R., \& Vink, J. S. 2006, A\&A, 460, L5

Kozyreva, A., Blinnikov, S., Langer, N., \& Yoon, S.-C. 2014, A\&A, 565, A70

Langer, N. 1997, in Astronomical Society of the Pacific Conference Series, Vol. 120, Luminous Blue Variables: Massive Stars in Transition, ed. A. Nota \& H. Lamers, 83

Langer, N. 1998, A\&A, 329, 551

-. 2012, ARA\&A, 50, 107

Langer, N., El Eid, M. F., \& Baraffe, I. 1989, A\&A, 224, L17

Li, W., Smith, N., Miller, A. A., \& Filippenko, A. V. 2009, The Astronomer's Telegram, 2212, 1

MacFadyen, A. I., \& Woosley, S. E. 1999, ApJ, 524, 262

Mackey, J., Mohamed, S., Gvaramadze, V. V., Kotak, R., Langer, N., Meyer, D. M.-A., Moriya, T. J., \& Neilson, H. R. 2014, Nature, 512, 282

Margutti, R., et al. 2014, ApJ, 780, 21

Mauerhan, J. C., et al. 2013, MNRAS, 430, 1801

Maza, J., et al. 2009, Central Bureau Electronic Telegrams, 1928, 1

Mazzali, P. A., Maurer, I., Valenti, S., Kotak, R., \& Hunter, D. 2010 MNRAS, 408, 87

Mazzali, P. A., Sauer, D. N., Pastorello, A., Benetti, S., \& Hillebrandt, W. 2008, MNRAS, 386, 1897

Mehner, A., Ishibashi, K., Whitelock, P., Nagayama, T., Feast, M., van Wyk, F., \& de Wit, W.-J. 2014, A\&A, 564, A14

Mestel, L. 1953, MNRAS, 113, 716

- 1957, ApJ, 126, 550

Mestel, L., \& Moss, D. L. 1986, MNRAS, 221, 25

Miller, A. A., Li, W., Nugent, P. E., Bloom, J. S., Filippenko, A. V., \& Merritt, A. T. 2009a, The Astronomer's Telegram, 2183, 1

Miller, A. A., et al. 2009b, ApJ, 690, 1303

Moriya, T., Tominaga, N., Tanaka, M., Maeda, K., \& Nomoto, K. 2010a, ApJ, 717, L83

Moriya, T., Tominaga, N., Tanaka, M., Nomoto, K., Sauer, D. N., Mazzali P. A., Maeda, K., \& Suzuki, T. 2010b, ApJ, 719, 1445

Moriya, T. J. 2014, A\&A, 564, A83

Moriya, T. J., Blinnikov, S. I., Baklanov, P. V., Sorokina, E. I., \& Dolgov, A. D. 2013, MNRAS, 430, 1402

Morris, T., \& Podsiadlowski, P. 2007, Science, 315, 1103

-. 2009, MNRAS, 399, 515

Müller, B., Janka, H.-T., \& Heger, A. 2012, ApJ, 761, 72

Nicholl, M., et al. 2013, Nature, 502, 346

Nieuwenhuijzen, H., \& de Jager, C. 1990, A\&A, 231, 134

O'Connor, E., \& Ott, C. D. 2011, ApJ, 730, 70

-. 2013, ApJ, 762, 126

Ofek, E. O., Lin, L., Kouveliotou, C., Younes, G., Göğüş, E., Kasliwal, M. M., \& Cao, Y. 2013a, ApJ, 768, 47

Ofek, E. O., et al. 2007, ApJ, 659, L13

-. 2013b, Nature, 494, 65

-. 2014, ApJ, 789, 104

Owocki, S. P. 2014, ArXiv e-prints: 1403.6745

Pan, T., Loeb, A., \& Kasen, D. 2012, MNRAS, 423, 2203

Pasquali, A., Nota, A., Langer, N., Schulte-Ladbeck, R. E., \& Clampin, M. 2000, AJ, 119, 1352

Paxton, B., Bildsten, L., Dotter, A., Herwig, F., Lesaffre, P., \& Timmes, F. 2011, ApJS, 192, 3

Paxton, B., et al. 2013, ApJS, 208, 4

Perets, H. B., \& Fabrycky, D. C. 2009, ApJ, 697, 1048

Podsiadlowski, P. 1992, PASP, 104, 717

—. 2010, New A Rev., 54, 39

Podsiadlowski, P., Fabian, A. C., \& Stevens, I. R. 1991, Nature, 354, 43

Podsiadlowski, P., \& Joss, P. C. 1989, Nature, 338, 401

Podsiadlowski, P., Joss, P. C., \& Hsu, J. J. L. 1992, ApJ, 391, 246

Podsiadlowski, P., Joss, P. C., \& Rappaport, S. 1990, A\&A, 227, L9

Podsiadlowski, P., Langer, N., Poelarends, A. J. T., Rappaport, S., Heger, A., \& Pfahl, E. 2004, ApJ, 612, 1044

Podsiadlowski, P., Morris, T. S., \& Ivanova, N. 2006, in Astronomical Society of the Pacific Conference Series, Vol. 355, Stars with the B[e]

Phenomenon, ed. M. Kraus \& A. S. Miroshnichenko, 259
Poelarends, A. J. T., Herwig, F., Langer, N., \& Heger, A. 2008, ApJ, 675, 614

Pols, O. R. 1994, A\&A, 290, 119

Pols, O. R., Schroder, K., Hurley, J. R., Tout, C. A., \& Eggleton, P. P. 1998, MNRAS, 298, 525

Pols, O. R., Tout, C. A., Eggleton, P. P., \& Han, Z. 1995, MNRAS, 274, 964

Portegies Zwart, S. F., \& van den Heuvel, E. P. J. 2007, Nature, 450, 388

Puls, J., Vink, J. S., \& Najarro, F. 2008, A\&A Rev., 16, 209

Quataert, E., \& Shiode, J. 2012, MNRAS, 423, L92

Quimby, R. M., Aldering, G., Wheeler, J. C., Höflich, P., Akerlof, C. W., \&

Rykoff, E. S. 2007, ApJ, 668, L99

Rakavy, G., \& Shaviv, G. 1967, ApJ, 148, 803

Rauscher, T., Heger, A., Hoffman, R. D., \& Woosley, S. E. 2002, ApJ, 576, 323

Rest, A., et al. 2012, Nature, 482, 375

Saio, H., Georgy, C., \& Meynet, G. 2013, MNRAS, 433, 1246

Sana, H., et al. 2012, Science, 337, 444

-. 2013, A\&A, 550, A107

Schneider, F. R. N., et al. 2014, ApJ, 780, 117

Shaviv, N. J. 2001, ApJ, 549, 1093

Shiode, J. H., \& Quataert, E. 2014, ApJ, 780, 96

Smartt, S. J. 2009, ARA\&A, 47, 63

Smith, N., Chornock, R., Li, W., Ganeshalingam, M., Silverman, J. M.

Foley, R. J., Filippenko, A. V., \& Barth, A. J. 2008, ApJ, 686, 467

Smith, N., Mauerhan, J. C., \& Prieto, J. L. 2014, MNRAS, 438, 1191

Smith, N., \& McCray, R. 2007, ApJ, 671, L17

Smith, N., \& Owocki, S. P. 2006, ApJ, 645, L45

Smith, N., \& Tombleson, R. 2014, ArXiv e-prints: 1406.7431

Smith, N., Vink, J. S., \& de Koter, A. 2004, ApJ, 615, 475

Smith, N., et al. 2007, ApJ, 666, 1116

Soker, N., \& Kashi, A. 2013, ApJ, 764, L6

Sukhbold, T., \& Woosley, S. E. 2014, ApJ, 783, 10

Takiwaki, T., Kotake, K., \& Suwa, Y. 2014, ApJ, 786, 83

Tanaka, M., Moriya, T. J., Yoshida, N., \& Nomoto, K. 2012, MNRAS, 2797

Tauris, T. M., \& van den Heuvel, E. P. J. 2014, ApJ, 781, L13

Timmes, F. X., Woosley, S. E., \& Weaver, T. A. 1996, ApJ, 457, 834

Trundle, C., Kotak, R., Vink, J. S., \& Meikle, W. P. S. 2008, A\&A, 483, L47

Ugliano, M., Janka, H.-T., Marek, A., \& Arcones, A. 2012, ApJ, 757, 69

van den Heuvel, E. P. J., \& Portegies Zwart, S. F. 2013, ApJ, 779, 114

van Marle, A. J., Smith, N., Owocki, S. P., \& van Veelen, B. 2010, MNRAS, 407, 2305

Vanbeveren, D., Mennekens, N., Van Rensbergen, W., \& De Loore, C. 2013, A\&A, 552, A105

Vink, J. S. 2012, in Astrophysics and Space Science Library, Vol. 384, Astrophysics and Space Science Library, ed. K. Davidson \& R. M. Humphreys, 221

Vink, J. S., Brott, I., Gräfener, G., Langer, N., de Koter, A., \& Lennon, D. J. 2010, A\&A, 512, L7

Vink, J. S., \& de Koter, A. 2002, A\&A, 393, 543

Vink, J. S., de Koter, A., \& Lamers, H. J. G. L. M. 1999, A\&A, 350, 181

-. 2000, A\&A, 362, 295

-. 2001, A\&A, 369, 574

Waldman, R. 2008, ApJ, 685, 1103

Walmswell, J. J., \& Eldridge, J. J. 2012, MNRAS, 419, 2054

Weiss, A. 1989, ApJ, 339, 365

Wellstein, S., Langer, N., \& Braun, H. 2001, A\&A, 369, 939

Woosley, S. E. 1993, ApJ, 405, 273

—. 2010, ApJ, 719, L204

Woosley, S. E., Blinnikov, S., \& Heger, A. 2007, Nature, 450, 390

Woosley, S. E., \& Heger, A. 2006, ApJ, 637, 914

Woosley, S. E., \& Timmes, F. X. 1996, Nuclear Physics A, 606, 137

Woosley, S. E., \& Weaver, T. A. 1986, ARA\&A, 24, 205

Yoon, S.-C., \& Cantiello, M. 2010, ApJ, 717, L62

Yoon, S.-C., \& Langer, N. 2005, A\&A, 443, 643

Yoon, S.-C., Langer, N., \& Norman, C. 2006, A\&A, 460, 199 